\title{
Historia de la regresión del desmán ibérico Galemys pyrenaicus (É. Geoffroy Saint-Hilaire, 1811) en el Sistema Central (Península Ibérica)
}

\author{
History of the decline of the Iberian desman Galemys pyrenaicus (É. Geoffroy \\ Saint-Hilaire, 1811) in the Central System (Iberian Peninsula) \\ Muturluzeak, Galemys pyrenaicusek, Erdialdeko Sisteman (Iberiar penintsula) \\ izandako atzerapenaren historia (E. Geoffroy Saint-Hilaire, 1811).
}

\author{
Julio Gisbert ${ }^{1 *}$, Rosa García-Perea1 \\ ${ }_{1}^{1}$ GALEMIA, Apdo. 45, Hoyo de Manzanares, 28240 Madrid, España. \\ *Corresponding author: galemia@gmail.com
}

\begin{abstract}
RESUMEN
En el Sistema Central, se ha producido un proceso de regresión de las poblaciones de desmán ibérico Galemys pyrenaicus (É. Geoffroy Saint-Hilaire, 1811), probablemente iniciado alrededor de 1960, en el que han influido diversos factores, todos ellos relacionados con las actividades humanas. Entre 1995 y 1998, reunimos las primeras evidencias del declive del desmán en el sur de su área de distribución. A lo largo de 15 años, se han realizado prospecciones sistemáticas en el Sistema Central, desde la Sierra de Ayllón hasta la Serra da Estrela, en áreas donde la especie había sido citada en tiempos históricos y cuyas condiciones ambientales eran aparentemente idóneas. Los resultados de dichas prospecciones fueron negativos en todos los casos, excepto en tres puntos aislados. Se ha estimado que la distribución de la especie en el Sistema Central se ha reducido en un 97\%. Esta regresión se atribuye fundamentalmente a la expansión del visón americano, al mal uso del agua por parte del hombre y al establecimiento de barreras que han contribuido a la fragmentación de las poblaciones.
\end{abstract}

PALABRAS CLAVE: Galemys pyrenaicus, Regresión, Sistema Central.

\section{ABSTRACT}

The populations of the Iberian desman Galemys pyrenaicus (É. Geoffroy Saint-Hilaire, 1811) have suffered a decline in the Central System of the Iberian peninsula since 1960. Several factors have been involved in this process, all of them related to human activities. The first evidences of this decline were gathered in the southern border of its range, between 1995 and 1998. Systematic surveys have been conducted for 15 years in the Central System, from Ayllon to Serra da Estrela, in areas where the presence of the species was previously reported, and the environmental characteristics were apparently adequate. The results were negative in all cases, except in three isolated points. We estimate the decrease of the distribution of the species in the study area at $97 \%$. Evidences suggest that this decline is mainly due to the expansion of the American mink, misuse of freshwater, and establishment of barriers that have contributed to the fragmentation of population.

KEY WORDS: Galemys pyrenaicus, Decline, Central System.

\section{LABURPENA}

Erdialdeko Sisteman, Muturluzearen (É. Geoffroy Saint-Hilaire, 1811) populazioek atzerapen-prozesua izan dute seguru asko 1960. urte inguruan hasita. Horretan hainbat faktorek eragin du eta guztiak daude gizakiaren jarduerekin lotuta. 1995 eta 1998 artean bildu genituen banaketa eremuaren hegoaldean muturluzeak bizi zuen beherakadaren lehen ebidentziak. 15 urtez, Erdialdeko Sisteman azterketa sistematikoak egin dituzte (Ayllón mendizerratik hasi eta Serra da Estrelarainoko eremuan) antzinako garaietan espeziearen inguruko aipuak genituen guneetan eta, ustez, ingurumen-baldintzak egokiak ziren tokietan. Azterketa horien emaitzak negatiboak izan ziren kasu guztietan isolatutako hiru puntutan izan ezik. Erdialdeko Sisteman, espeziearen banaketa \% 97 gutxitu dela kalkulatu dute. Atzeraldi hori, batez ere, honako hauek eragin dute: bisoi amerikarraren hedapena, gizakiak ura gaizki erabiltzea eta populazioak haustea eragin duten barrerak ezarri izana.

GAKO-HITZAK: Galemys pyrenaicus, Atzeraldia, Erdialdeko Sistema.

\section{INTRODUCCIÓN}

El desmán ibérico Galemys pyrenaicus (É. Geoffroy Saint-Hilaire, 1811) es un mamífero Soricomorfo de la familia de los Tálpidos, endémico de la península Ibérica y de costumbres semiácuaticas.

En el pasado, hace 5.000-6.000 años, cuando la actividad humana no había producido aún gran impacto en las poblaciones animales (Owen-Smith, 1999; Ellis et al., 2013), probablemente el desmán ibérico abarcó todos los ríos de montaña de la mitad norte de la península lbérica, exceptuando el valle del Ebro y la costa mediterránea. En un periodo más reciente (siglo XX), la especie ocupaba los ríos de montaña del norte de Portugal, el Macizo Galaico, los Montes de León, la Cordillera Cantábrica, el Corredor Vasco, Pirineos, el Sistema Ibérico y el Sistema Central (Fig. 1), lo que se ha denominado el "Escenario Sigma", referente para futuros estudios de recuperación de la especie (Gisbert \& García-Perea, 2010d). Su área de distribución afecta, pues, a las administracio- 


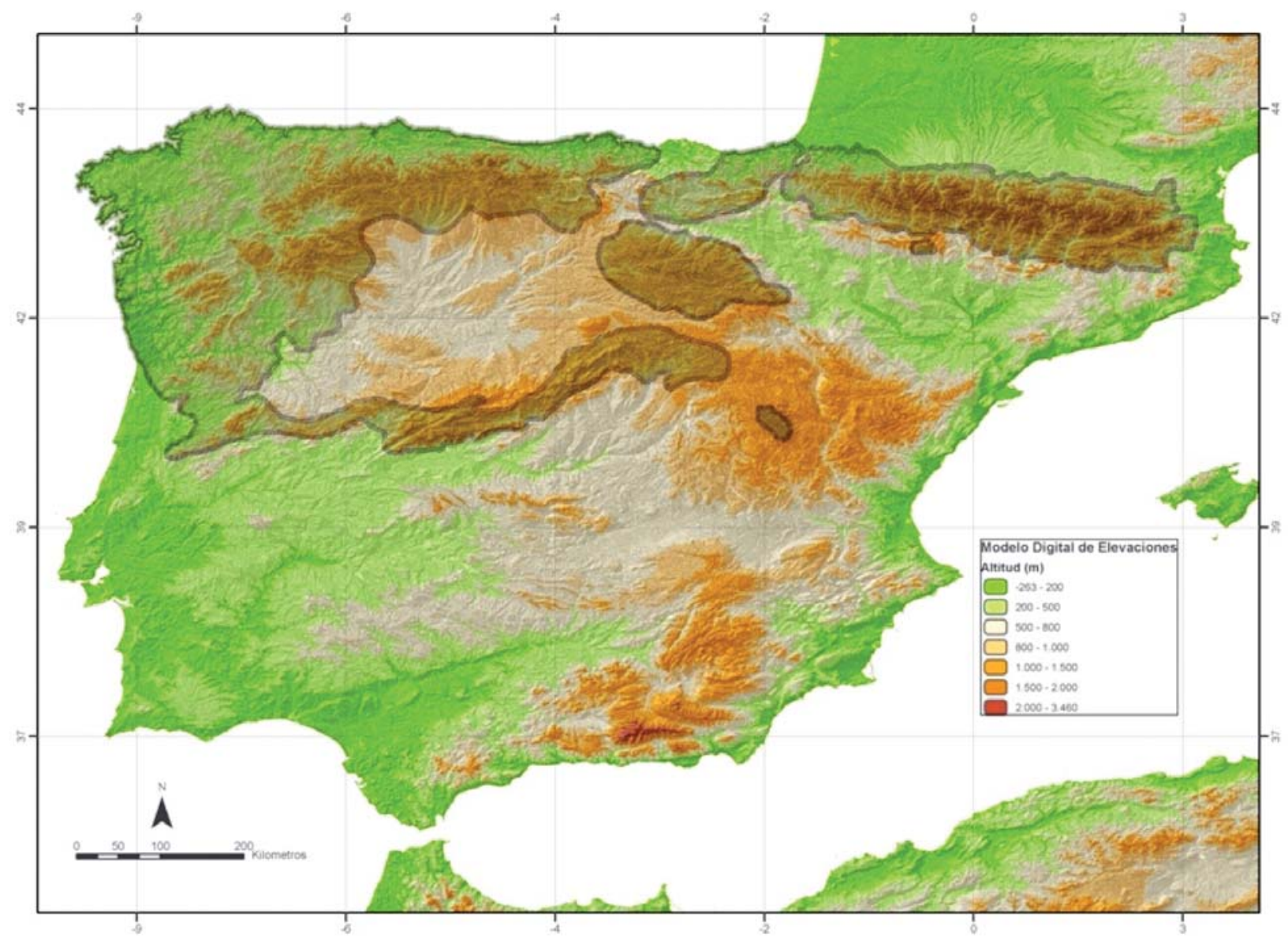

Fig. 1. - Escenario Sigma (Gisbert \& García-Perea, 2010d): área de distribución estimada del desmán ibérico en la península Ibérica en la década de los 90 , según la recopilación realizada por Nores (1992) y otros datos históricos disponibles.

Fig. 1. - Sigma scenario (Gisbert \& García-Perea, 2010d): estimated distribution range of Iberian desman in the Iberian Peninsula, in the decade of the 90s, based on Nores (1992) and other historical data available.

nes de cuatro países: España, Portugal, Francia y Andorra (Nores et al., 2002, 2007; Gisbert \& García-Perea, 2010c). Existen citas fósiles de desmanes en el sur de la Península, atribuibles a otra especie: Galemys kormosi (Rumke, 1985), del Pleistoceno temprano.

El desmán ibérico se encuentra en la actualidad amenazado en toda su área de distribución, figurando en el Catálogo Español de Especies Amenazadas como Vulnerable, con el matiz de En Peligro de Extinción en el Sistema Central (R.D. 139/2011: M.A.R.M., 2011). Pero esta situación no se ha reconocido hasta muy recientemente en España (Nores, 2007), fundamentalmente debido a los escasos estudios realizados sobre la especie, tanto por la dificultad de los trabajos de inventario, como por la escasez de fondos dedicados a su conservación.

El desmán ibérico ha sido conocido desde antiguo en el Sistema Central por sus lugareños, en especial aquellos relacionados con los ríos. Antiguas profesiones, como la de pescador fluvial, molinero o regante, obligaban en el pasado a invertir largas jornadas en los ríos de montaña. Asimismo, el abastecimiento diario de agua para los habitantes y para sus ganados, así como la necesidad de lavar en el río, favorecían el conocimiento del medio fluvial en los pueblos ribereños. Durante el desarrollo de estas actividades, se producían los avistamientos de esta especie, que era conocida hasta el punto de tener nombre vernáculo propio desde antiguo: almizclera (Graells, 1852, 1897; Martínez y Sáez, 1876; Cabrera, 1914; De la Fuente Arrimadas, 1926). Esta especie ya era conocida en las Sierras de Gredos por los pescadores profesionales de trucha, en las décadas de 1960 y 1970, como pudimos comprobar personalmente a partir de los años 70 (J. Gisbert). Sus técnicas de pesca incluían además de la caña, el maneo, es decir, atrapar a mano las truchas ocultas debajo de las piedras, por lo que con cierta frecuencia eran mordidos por el desmán. También usaban garlitos de mimbre (nasas) para la captura de truchas Salmo trutta Linnaeus, 1758, en las que ocasionalmente aparecían desmanes ahogados.

Por estos motivos, los lugareños conocían perfectamente la morfología del desmán, y cuando lo sacaban de las nasas, o se lo encontraban durante los maneos, no lo confundían con otras especies de mamíferos de hábitos semiacuáticos, como el musgaño de Cabrera Neomys anomalus Cabrera, 1907 o la rata de agua Arvicola sapidus Miller, 1908. También los molineros co- 
nocían el desmán, ya que con frecuencia se introducían por la acequia del molino y morían atrapados entre los peines de los canales de entrada de agua. Los guardas de pesca de la zona conocieron esta especie desde antiguo, vinculada al hábitat de las truchas. El desmán ha estado también presente en el folklore regional. Por ejemplo, en el valle del alto Tormes (vertiente norte de las Sierras de Gredos) existía en el pasado la costumbre de incluir pieles de esta especie entre el ajuar de las novias para evitar el ataque de las polillas y perfumar los tejidos. Las almizcleras, cuyo nombre hace referencia precisamente al intenso olor del almizcle producido por sus glándulas anales, debían ser capturadas por los mozos a mano, como acto de cierta valentía, ya que cuando los desmanes muerden producen a veces heridas aparatosas, debido a sus incisivos biselados. Áurea Gargantilla, vecina de La Angostura (Ávila), nos relató en 1976 esta curiosa costumbre que ella conoció de joven, añadiendo que existían almizcleras de oro y de plata, refiriéndose sin duda a las irisaciones doradas o plateadas del pelaje que presenta esta especie dentro del agua (Gisbert et al., 2000).

En este artículo se revisan los datos históricos disponibles sobre la distribución y regresión del desmán ibérico en el Sistema Central, así como los factores que se considera que han intervenido en dicha regresión, con la intención de que esta información sea útil a la hora de aplicar posteriormente los planes de conservación y restauración de sus poblaciones.

\section{MATERIAL Y MÉTODOS}

\section{Área de estudio}

El área de estudio abarca todo el Sistema Central, desde la Sierra de Ayllón en el este, hasta la Serra da Estrela (Portugal) en el oeste (Fig. 2). Parte de los datos apor- tados en este artículo se recogieron durante la década de los 70, y otra parte corresponde al período 1995-2011.

\section{Metodología para determinar la distribución histórica del desmán en el Sistema Central}

Para determinar el área de distribución histórica del desmán, se han utilizado dos fuentes de datos. Por un lado, se ha realizado una recopilación de citas de presencia de la especie (ejemplares depositados en diferentes museos y colecciones científicas, citas bibliográficas de autores fiables) y citas de su ausencia (prospecciones realizadas por profesionales fiables). Asimismo, se han filtrado los datos asociados a las citas, en especial la fecha de captura u observación (no la fecha de publicación del artículo, o del informe) y la localización exacta del punto de captura (las citas son a veces poco precisas, refiriéndose a un municipio por cuyo territorio pasan diversos ríos o arroyos). Determinar la autoría de una cita que ha sido recogida por diversos autores en diferentes artículos e informes, es también una labor importante para evitar repeticiones de la misma, o buscar la información en la fuente original. Los datos de los ejemplares depositados en colecciones científicas, tanto españolas como extranjeras, son especialmente valiosos, pues cada ejemplar es prueba inequívoca de la presencia del desmán en la localidad correspondiente, y en la fecha indicada en su documentación.

La segunda fuente de datos proviene de los muestreos realizados en diferentes áreas del Sistema Central, entre 1995 y 2011. Estos muestreos no han sido sistemáticos, ya que han dependido de la sensibilidad de las distintas administraciones autonómicas hacia la situación de la especie, así como de los fondos disponibles.

En dichos muestreos se han utilizado nasas ligeras de tensión continua (especialmente diseñadas por los auto-

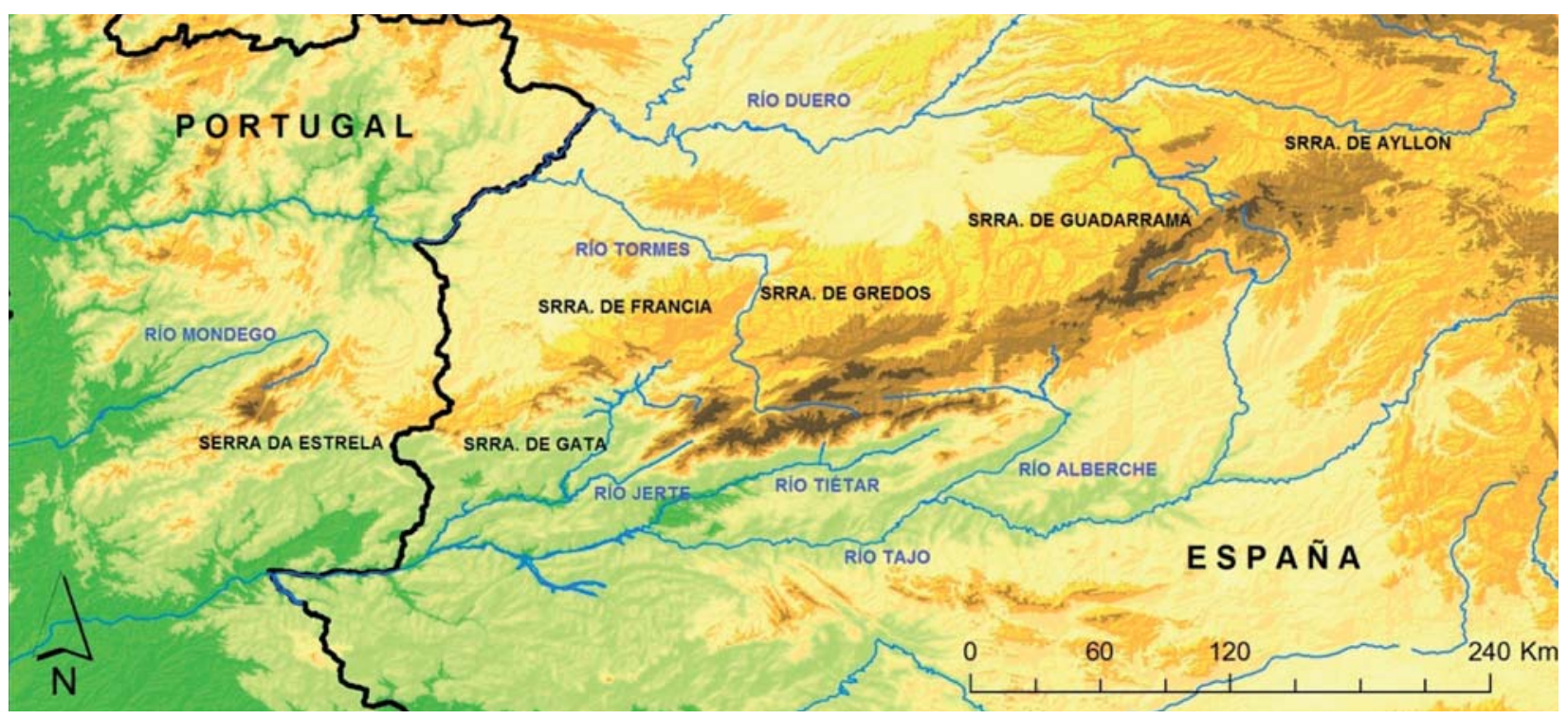

Fig. 2. - Localización de las diferentes áreas del Sistema Central que se citan en este artículo.

Fig. 2. - Location of the different areas of the Central System mentioned in this article. 
res), según el método propuesto por Richard (1973) y mejorado por Nores (1992) y Bertrand (1992). Se han colocado unas 20-30 nasas cada $150 \mathrm{~m}$ de río, con la boca dirigida en sentido de la corriente, durante 3 días y 2 noches, realizando revisiones cada 1 o 2 horas. En total, se ha realizado un esfuerzo de muestreo de 3495 nasas/24 h (Tabla 1). También se han realizado búsquedas de excrementos de desmán, según el método propuesto por Queiroz (1991), Nores (1992), Bertrand (1992), y mejorado por Aymerich et al. (2001) y Fernández González (2007). Este método consiste en inspeccionar oquedades y piedras a lo largo de un tramo de 250-500 m. Complementariamente, se han recogido excrementos de nutria Lutra lutra (Linnaeus, 1758) y visón americano Neovison vison (Schreber, 1777), con el objeto de buscar posibles restos de desmán en ellos, un método efectivo, de gran utilidad para estos propósitos, como describe Romero $(2007,2010)$ con materiales de Galicia.

Todas las citas obtenidas se han georeferenciado y proyectado en mapas físicos, obteniéndose así una distribución temporal de dichas citas.

\section{RESULTADOS Y DISCUSIÓN}

La búsqueda y filtrado de datos ha arrojado un número elevado, pero indeterminado, de citas de la espe- cie, ya que las citas históricas más antiguas se refieren a menudo a "múltiples observaciones", o "presencia frecuente" del desmán, en localidades a veces tan amplias como "Sierra de Gredos". En las Tablas 2 y 3 se detallan, respectivamente, las citas positivas y negativas recopiladas, indicando los autores y el periodo temporal correspondiente, así como, en su caso, el museo donde se encuentra el ejemplar.

Interesa mencionar que, hasta 1992, los datos existentes sobre la distribución del desmán se refieren fundamentalmente a su presencia, basada en observaciones puntuales. Asimismo, de dicho periodo no se dispone prácticamente de datos de ausencia, ya que no se han realizado prospecciones sistemáticas en zonas amplias utilizando métodos de captura o de detección de sus excrementos que hayan arrojado resultados negativos. Sin embargo, durante las dos últimas décadas sí se han realizado muestreos específicos para delimitar el área de distribución de la especie en el Sistema Central, además de recogerse citas puntuales de presencia (Tablas 2 y 3 ).

El mapa de la Fig. 3 muestra todas las localidades donde se ha citado la presencia de la especie en el Sistema Central.

\begin{tabular}{|l|c|c|c|}
\hline \multicolumn{1}{|c|}{ Autores } & N tramos o ríos & Esfuerzo de muestreo & Distancia muestreada \\
\hline Gisbert \& Fernández-Salvador (1998) & 10 & 462 nasas/24h & $9500 \mathrm{~m}$ \\
\hline Gisbert et al. (2000) & 12 & 304 nasas/24h & $7750 \mathrm{~m}$ \\
\hline García-Perea et al. (2001) & 13 & 84 nasas/24h & $3220 \mathrm{~m}$ \\
\hline Gisbert \& García-Perea (2004a) & 26 & 1114 nasas/24h & $11870 \mathrm{~m}$ \\
\hline Gisbert \& García-Perea (2004b) & 25 & 1332 nasas/24h & $3498 \mathrm{~m}$ \\
\hline Gisbert \& García-Perea (2011) & 1 & 20 nasas/24h & $100 \mathrm{~m}$ \\
\hline TOTAL & $\mathbf{8 9}$ & $\mathbf{3 4 9 5}$ nasas/24h & $\mathbf{3 5 9 3 8} \mathbf{~ m}$ \\
\hline
\end{tabular}

Tabla 1. - Esfuerzos de muestreo realizados en los distintos estudios prospectivos que ha desarrollado nuestro equipo durante los últimos 15 años en el Sistema Central. Table 1. Sampling efforts invested in the different surveys carried out by our team over the last 15 years in the Central System.

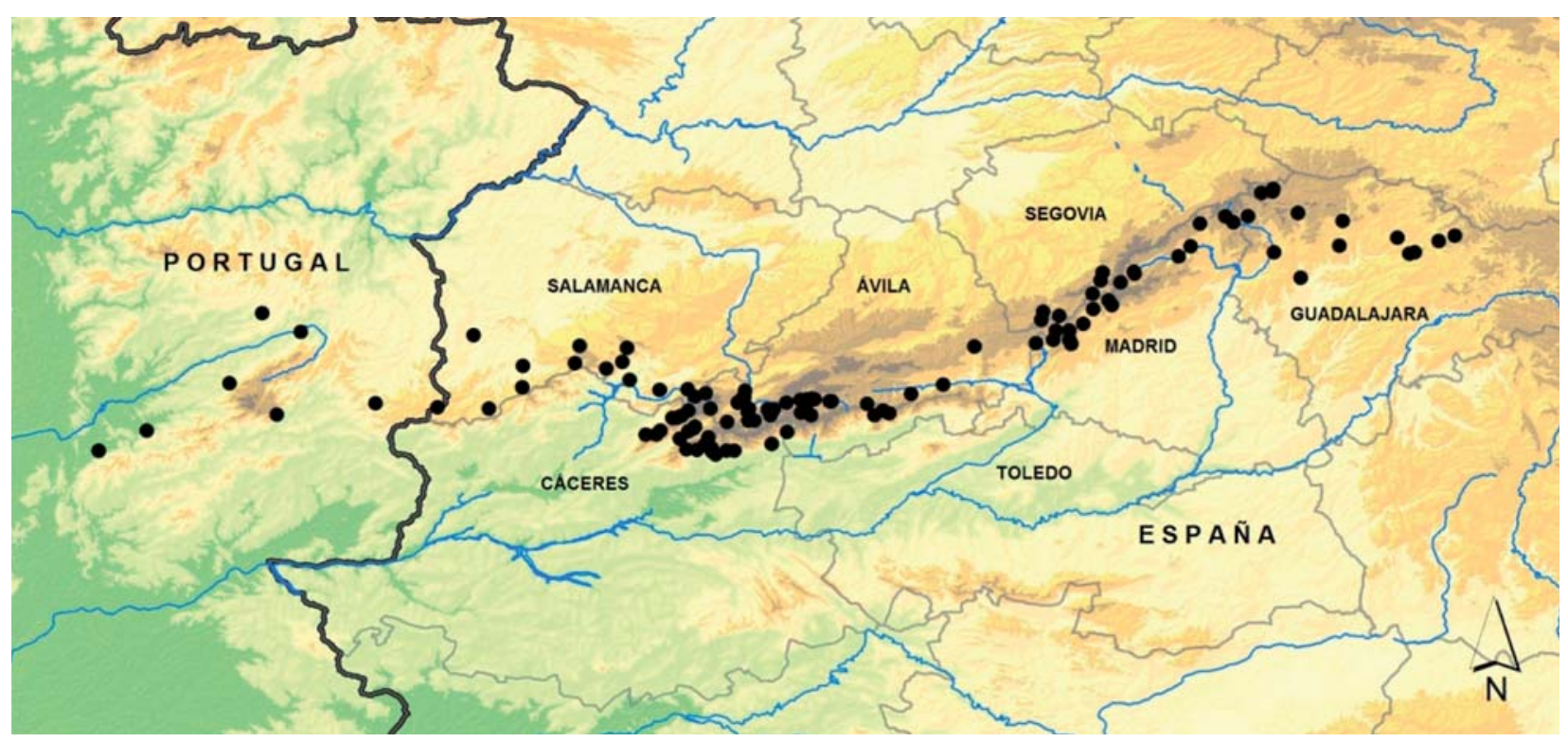

Fig. 3. - Círculos negros: todas las localidades con citas de presencia de desmán ibérico en el Sistema Central.

Fig. 3. - Black circles: all the localities with presence reported of Iberian desman in the Central System. 


\begin{tabular}{|c|c|c|c|c|}
\hline Localidad / Río & Término Municipal & Fecha & Fuente & Tipo de cita \\
\hline Todo el Sistema Central & & 1914 & Cabrera (1914) & MOC \\
\hline Sierras de Gredos & & $<1847$ & Graells (1897) & MOC \\
\hline Sierras de Gredos & & 1876 & Martínez y Sáez (1876) & $3 \mathrm{C}$ \\
\hline Sierras de Gredos & & 1972 & Rey (1972) & MOC \\
\hline Sierras de Gredos & & 1977 & Rey y Martínez-Rica (1977) & MOC \\
\hline Sierras de Gredos & & 1983 & Gisbert (1983) & MOC \\
\hline Sierras de Gredos & & 1988 & Gisbert y García-Perea (1988) & MOC \\
\hline \multicolumn{5}{|c|}{ GUADALAJARA (ESPAÑA) } \\
\hline Río Bornova & S. Andrés del Congosto & 1970 & J.A. Saboya, en Gisbert y Fdez-Salvador (1998) & 0 \\
\hline Río Jarama & El Cardoso de la Sierra, Sa de Ayllón & 1970 & J. Garzón, en Nores (1992) & \\
\hline Río Bornova & Hiendelaencina & 1972 & J. Martín, en Gisbert y Fdez-Salvador (1998) & 0 \\
\hline Río Dulce & Pelegrina & 1974 & Pérez Lucas en Gisbert y Fdez-Salvador (1998) & $2 \mathrm{C}$ \\
\hline Río Dulce & Aragosa & 1975 & A. Pérez, en Gisbert y Fdez-Salvador (1998) & C \\
\hline Río Dulce & Mandayona & 1975 & A. Pérez y J. Lobón, en Nores (1992) & $2 \mathrm{C}$ \\
\hline Río Lillas & Cantalojas, $S^{\underline{a}}$ de Ayllón & $1973,78,92$ & MNCN-EBD & $4 \mathrm{C}$ \\
\hline Río Salado & Viana de Jadraque & 1982 & J. Lobón, Nores (1992) & C \\
\hline Río Saúca & Saúca & 1982 & J. Lobón, Nores (1992) & C \\
\hline Río Sorbe & Razbona & 1988 & Querol, en Nores (1992) & \\
\hline Río de la Hoz & La Ponvieja & 1988 & G. Cerezo, en Gisbert y Fdez-Salvador (1998) & 0 \\
\hline Río Jarama & Monasterio Bonaval, Retiendas & 1989 & G. García, en Gisbert y Fdez-Salvador (1998) & 0 \\
\hline Río Berbellido & Bocígano, Sạ de Ayllón & 1992 & F. Asenjo, en Gisbert y Fdez-Salvador (1998) & 0 \\
\hline Río Zarza & Cantalojas, $\mathrm{S}^{\mathrm{a}}$ de Ayllón & 1992 & P. Cerezo, en Gisbert y Fdez-Salvador (1998) & 0 \\
\hline Río Sorbe & Umbralejo & 1996 & Gisbert y Fernández-Salvador (1998) & 0 \\
\hline \multicolumn{5}{|c|}{ SEGOVIA (ESPAÑA) } \\
\hline Rio Eresma & Navacerrada hasta S. Ildefonso & 1847 & Graells (1897) & MOC \\
\hline \multirow[t]{4}{*}{ Rio Valsaín } & Venta los Mosquitos S. Ildefonso & 1847 & Graells (1897) & C \\
\hline & Puerto de La Fuenfría & 1847 & Graells (1897) & MOC \\
\hline & San Rafael & 1847 & Graells (1897) & MOC \\
\hline & El Espinar & 1847 & Graells (1897) & MOC \\
\hline Arroyo del Boquerón & Boca del Infierno, El Espinar & 1847 & Graells (1897) & MOC \\
\hline \multirow{2}{*}{ Río Eresma } & Valsaín & 1912 & Cabrera (1912) & \\
\hline & Segovia, Sierra de Guadarrama & 1927 & MNCN & C \\
\hline Río Pirón & Nacimiento del río & 1989 & Nores (1992) & 0 \\
\hline Río Acebedas & Revenga & 2000 & García \& Lizana (2007) & 0 \\
\hline \multicolumn{5}{|l|}{ MADRID (ESPAÑA) } \\
\hline & El Escorial & 1838 & Graells (1897) & C \\
\hline Arroyos de Siete Picos & & 1847 & Graells (1897) & MOC \\
\hline Arroyos Pico Maliciosa & Becerril de la Sierra & 1847 & Graells (1897) & MOC \\
\hline Río Guadarrama & Guadarrama & 1847 & Graells (1897) & MOC \\
\hline Arroyo Cuelgamuros & Valle de Cuelgamuros & 1847 & Graells (1897) & MOC \\
\hline \multirow[t]{2}{*}{ Río Lozoya } & Valle Lozoya Peñalara-Buitrago & 1847 & Graells (1897) & MOC \\
\hline & El Escorial & 1850 & Graells (1852-1858) & C \\
\hline Arroyo del Plantel & presa El Romeral, S.L. Escorial & 1850 & Graells (1852-1858) & $\mathrm{O}$ \\
\hline Fuente de La Teja & S. Lorenzo del Escorial & 1850 & Graells (1852-1858) & 0 \\
\hline Río Lozoya & Buitrago de Lozoya & antes de & Miller (1912), USNM & C \\
\hline Río Lozoya & Buitrago de Lozoya & 1912 & Cabrera (1914), MNCN & $\mathrm{C}$ \\
\hline Río Lozoya & Garganta de los Montes & 1914 & Cabrera (1912), MNCN & $3 \mathrm{C}$ \\
\hline Arroyo Garcisancho & La Umbría, Rascafría & 1912 & J. Arribas, en Nores (1992) & \\
\hline El Ventorrillo & Navacerrada & 1970 & V. Pérez Mellado, en Nores (1992) & \\
\hline Río Madarquillos & Coto de Horcajo, H. de la Sierra & 1971 & Hernán, en Nores (1992) & \\
\hline Río de la Angostura & El Paular & 1981 & G. Muñoz, en García-Perea et al. (2001) & 0 \\
\hline Río Aceña & Sta. María de la Alameda & 1982 & E. Virgós (com. pers.) & 0 \\
\hline Arroyo Peñalara & Rascafría & 1990 & E. Virgós (com. pers.) & 0 \\
\hline Río Jarama & Hayedo de Montejo & 1990 & L. de la Cuesta, en Nores (1992) & $\mathrm{O}$ \\
\hline \multicolumn{5}{|l|}{ ÁVILA (ESPAÑA) } \\
\hline Rio Eresma & Vertiente norte de Gredos & 1976 & J. Gisbert y J. Melendro (1976) & MOC \\
\hline Rio Valsaín & Sierra del Barco & 1847 & Graells (1897) & $\mathrm{MOC}$ \\
\hline
\end{tabular}




\begin{tabular}{|c|c|c|c|c|}
\hline \multicolumn{5}{|c|}{ ÁVILA (ESPAÑA) continuación } \\
\hline & Sierra de la Duquesa & 1847 & Graells (1897) & MOC \\
\hline & Sierra de Tornavacas & 1847 & Graells (1897) & $\mathrm{MOC}$ \\
\hline & Sierra de Ávila & 1847 & Graells (1897) & $\mathrm{MOC}$ \\
\hline & Las Navas del Marqués & 1847 & Graells (1897) & MOC \\
\hline & Navaluenga & 1847 & Graells (1897) & $\mathrm{MOC}$ \\
\hline & Puerto del Pico & 1847 & Graells (1897) & MOC \\
\hline & Peguerinos & 1897 & Graells (1897) & $\mathrm{C}$ \\
\hline & Villanueva de Ávila & 1908 & F. Arias (1908), MNCN & C \\
\hline & El Herradón & 1912 & Cabrera (1912), MNCN & C \\
\hline & El Barco de Ávila & 1926 & De la Fuente Arrimadas (1926) & $\mathrm{O}$ \\
\hline Río Tormes & Navalperal de Tormes, $S^{\underline{a}}$ Gredos & 1931 & MNCN & $2 \mathrm{C}$ \\
\hline Río Tormes & San Juan de Gredos, Sª Gredos & 1935 & R. Núñez (1935), MNCN & C \\
\hline Río Gaznata & Herradón de Pinares & 1935 & MNCN & C \\
\hline Charco de Peñagorda & El Barco de Ávila, S ${ }^{a}$ Gredos & década 1950 & F. Rodriguez Vaquero, en Gisbert et al. (2000) & $\mathrm{O}$ \\
\hline Ggta. de Navamediana & Navamediana, $S^{\mathrm{a}}$ Gredos & década 1950 & H. García, en Gisbert et al. (2000) & MC \\
\hline Garganta de Bohoyo & Bohoyo, $S^{\mathrm{a}}$ Gredos & década 1950 & H. García, en Gisbert et al. (2000) & MC \\
\hline Río Aravalle & hasta el Tormes El Barco de Ávila & década 1960 & F. Rodriguez Vaquero, en Gisbert et al. (2000) & $\mathrm{MO}$ \\
\hline Río Aravalle & El Barco de Ávila & 1960 & D. Parrón, en Gisbert et al. (2000) & $\mathrm{C}$ \\
\hline Río Tormes & Navalperal de Tormes, $S^{a}$ Gredos & 1969 & G. Niethammer (1970), AKM & $10 \mathrm{C}$ \\
\hline Ggta. de los Caballeros & Navamures & década 1970 & C. Mañoso, en Gisbert et al. (2000) & $\mathrm{MO}$ \\
\hline Río Tormes & Presa de Navamorisca & 1970 & A. Parrón, en Gisbert et al. (2000) & $\mathrm{O}$ \\
\hline Río Tormes & Navalperal de Tormes & 1973 & A. Salvador, en Gisbert et al. (2000) & EM \\
\hline Río Tormes & Molino de, Hoyos del Espino & 1974 & J. Gisbert y J. Melendro, en Gisbert et al. (2000) & C \\
\hline Río Malillo & El Chorro, Solana de Ávila & 1974 & J. Gisbert y J. Melendro, en Gisbert et al. (2000) & $\mathrm{C}$ \\
\hline Río Aravalle & La Carrera & 1974 & J. Gisbert y J. Melendro, en Gisbert et al. (2000) & C \\
\hline Garganta de Gredos & Navalperal de Tormes & 1974 & J. Gisbert y J. Melendro, en Gisbert et al. (2000) & C \\
\hline Río Tormes & Charco del Churrero, Barco de Ávila & 1975 & D. Parrón, en Gisbert et al. (2000) & 0 \\
\hline Garganta de la Vega & La Escaruela, Puerto Castilla & 1975 & J. Gisbert y J. Melendro, en Gisbert et al. (2000) & $\mathrm{C}$ \\
\hline Ggta. de los Caballeros & Molino de Tormellas & 1976 & J. Gisbert y J. Melendro, en Gisbert et al. (2000) & EM \\
\hline Ggta. de los Caballeros & Navalguijo & 1976 & J. Gisbert y J. Melendro, en Gisbert et al. (2000) & $\mathrm{C}$ \\
\hline Río Tormes & La Angostura, Zapardiel Ribera & 1976 & A. Prieto Gargantilla, en Gisbert et al. (2000) & EM \\
\hline Garganta Tejea & Majada del Pinillo, El Raso & 1976 & J. Gisbert y J. Melendro, en Gisbert et al. (2000) & C \\
\hline Garganta de Bohoyo & Mata Asnos, Bohoyo & 1976 & J. Gisbert y J. Melendro, en Gisbert et al. (2000) & $E G$ \\
\hline Garganta Berrocosa & Navalonguilla & 1977 & J. Gisbert y J. Melendro, en Gisbert et al. (2000) & $E G$ \\
\hline Río Tormes & molino de Hoyos del Espino & 1980 & B. Ramos (1980), MNCN-EBD & C \\
\hline Río Tormes & molino de Hoyos del Espino & 1980 & B. Ramos (1980), MNCN-EBD & C \\
\hline Garganta del Pinar & Navalperal de Tormes & 1991 & F. García, en Gisbert et al. (2000) & 0 \\
\hline Arroyo Suarina & San Esteban del Valle & 1992 & Nores (1992) & $\mathrm{O}$ \\
\hline Ggta. de los Caballeros & Navalonguilla & 1992 & García-Díaz (2012) & EM \\
\hline Garganta del Cervunal & Laguna del Cervunal, Navalperal & 1995 & A. Parrón, en Gisbert et al. (2000) & $\mathrm{C}$ \\
\hline Garganta de Eliza & Pedro Bernardo & 1995 & C. del Arco, en Gisbert et al. (2000) & $\mathrm{O}$ \\
\hline Garganta del Cervunal & Navalperal de Tormes & 2000 & Toro et al. (2001) & O \\
\hline \multicolumn{5}{|c|}{ CÁCERES (ESPAÑA) } \\
\hline Garganta de Minchones & & década 1980 & J. Trejo, en Gisbert y García-Perea (2004a) & $\mathrm{MO}$ \\
\hline Río Jerte & Jerte & 1980 & L. F. Estévez, en Gisbert y García-Perea (2004a) & EM \\
\hline \multirow[t]{2}{*}{ Río Caparro } & Casas del Monte & 1981 & J. Garzón, en Nores (1992) & 0 \\
\hline & Segura de Toro & 1982 & Arcas, en Nores (1992) & $\mathrm{O}$ \\
\hline Río Ambroz & Hervás & 1985 & J. Garzón, en Nores (1992) & 0 \\
\hline Garganta del Yedrón & Aldeanueva de la Vera & 1985 & A. Iglesias, en Malo de Molina y Solano (1989) & $\mathrm{C}$ \\
\hline Arroyo del Losar & El Losar de la Vera & 1987 & A. Iglesias, en Malo de Molina y Solano (1989) & 0 \\
\hline Garganta Jaranda & Jarandilla de la Vera & 1988 & J. Garzón, en Nores (1992) & 0 \\
\hline Río Jerte & Cabezuela del Valle & 1988 & J. Garzón, en Nores (1992) & 0 \\
\hline \multirow[t]{2}{*}{ Garganta Jaranda } & Puente de Jarandilla de la Vera & 1988 & Pescador, en Gisbert y García-Perea (2004a) & $\mathrm{C}$ \\
\hline & Casas del Monte & 1989 & Malo de Molina y Solano (1989) & C \\
\hline Río Ambroz & Piscifactoría de Hervás, Hervás & 1989 & Malo de Molina y Solano (1989) & C \\
\hline Río Jerte & Jerte & 1989 & F. Prieto, en Malo de Molina y Solano (1989) & $\mathrm{C}$ \\
\hline Garganta Jaranda & Jarandilla de la Vera & 1989 & F. Prieto, en Malo de Molina y Solano (1989) & $\mathrm{C}$ \\
\hline Río Jerte & Cabezuela del Valle & 1991 & J.A. Torres, en Gisbert y García-Perea (2004a) & $\mathrm{C}$ \\
\hline
\end{tabular}




\begin{tabular}{|c|c|c|c|c|}
\hline \multicolumn{5}{|c|}{ CÁCERES (ESPAÑA) continuación } \\
\hline Río Vadillo & El Losar de la Vera & 1992 & Nores (1992) & \\
\hline Garganta del Hornillo & Tornavacas & 1992 & Agente M.A. en Gisbert y García-Perea (2004a) & 0 \\
\hline Río Ambroz & Piscifactoría de Hervás, Hervás & 2000 & Encargado, en Gisbert y García-Perea (2004a) (2004a) & EM \\
\hline Garganta Mayor & Garganta la Olla & 2000 & A. Díaz, en Gisbert y García-Perea (2004a) & 0 \\
\hline Garganta de Cuartos & El Losar de la Vera & 2001 & Agente M.A. en Gisbert y García-Perea (2004a) & 0 \\
\hline Río Ambroz & Hervás & 2003 & Gisbert y García-Perea (2004a) & $2 \mathrm{C}$ \\
\hline Chorrera de la Mora & Guachos, Aldeanueva de la Vera & 2003 & Gisbert y García-Perea (2004a) & C \\
\hline Río Ambroz & Hervás & 2003 & Gisbert y García-Perea (2004b) & $12 \mathrm{C}$ \\
\hline Río Ambroz & Hervás & 2011 & Gisbert y García-Perea (2011) & $\mathrm{C}+\mathrm{IG}$ \\
\hline \multicolumn{5}{|c|}{ SALAMANCA (ESPAÑA) } \\
\hline Río de Candelario & Candelario & 1977 & J. Gisbert y J. Melendro & $\mathrm{C}$ \\
\hline Río Cuerpo de Hombre & Béjar, S- de Gredos & 1975 & Sans Coma, EBD & $2 \mathrm{C}$ \\
\hline Arroyo del Oso & La Cobatilla & 1976 & J. Gisbert y J. Melendro (1976) & C \\
\hline Río Cuerpo de Hombre & Lagunilla & 1956 & J. Niethammer (1956) & C \\
\hline Río Cuerpo de Hombre & Lagunilla & 1964 & J. Niethammer (1964) & C \\
\hline Río Alagón & Sotoserrano & 1978 & V. Pérez-Mellado, en Nores (1992) & \\
\hline Río Águeda & Gallegos de Argañán & 1992 & Martínez de Albéniz, en Nores (1992) & EG \\
\hline Río Francia & El Caserito & 1996 & Morales et al. (2004) & LU \\
\hline Río Francia & Casas del Conde & 1996 & Morales et al. (2004) & LU \\
\hline Río Cuerpo de Hombre & Hoya Moros & 1997 & Bueno (1998) & $\mathrm{O}$ \\
\hline Río Cuerpo de Hombre & Navasfrías & 1999 & Peris et al. (1999) & \\
\hline \multirow[t]{5}{*}{ Río Frío } & Puente Perales, Villasrubias & 1999 & Peris et al. (1999) & \\
\hline & Zamarra & 1999 & Peris et al. (1999) & \\
\hline & Agallas & 1999 & Peris et al. (1999) & \\
\hline & Monsagro & 1999 & Peris et al. (1999) & \\
\hline & El Maíllo & 1999 & Peris et al. (1999) & \\
\hline Río Francia & El Caserito & 1999 & Peris et al. (1999) & $2 \mathrm{CT}$ \\
\hline \multicolumn{5}{|c|}{ SERRA DA ESTRELA (PORTUGAL) } \\
\hline & Sabugueiro de Guarda & 1972 & Engels (1972) & 0 \\
\hline Ríos Zezere y Caria & Covilha de Castelo Branco & 1989 & Queiroz (1989) & $\mathrm{C}+\mathrm{EX}$ \\
\hline Río Mondego & Celorico de Beira, Guarda & 1989 & Queiroz (1989) & $\mathrm{C}+\mathrm{EX}$ \\
\hline Río Seia & Seia, Guarda & 1989 & Queiroz (1989) & $\mathrm{C}+\mathrm{EX}$ \\
\hline Río Coa & Sabugal, Guarda & 1989 & Queiroz (1989) & $\mathrm{C}+\mathrm{EX}$ \\
\hline Río Alva & Arganil, Coimbra & 1989 & Queiroz (1989) & $\mathrm{C}+\mathrm{EX}$ \\
\hline Río Ceira & Lousa, Guarda & 1989 & Queiroz (1989) & $\mathrm{C}+\mathrm{EX}$ \\
\hline Río Ceira & Pampilhosa da Serra, Guarda & 1989 & Queiroz (1989) & $\mathrm{C}+\mathrm{EX}$ \\
\hline Río Meimoa & Penamacor, Castelo Branco & 1989 & Queiroz (1989) & $\mathrm{C}+\mathrm{EX}$ \\
\hline
\end{tabular}

Tabla 2. - Citas de presencia de desmán ibérico en el Sistema Central. Colecciones donde se encuentran depositados los ejemplares citados: EBD (Estación Biológica de Doñana, Sevilla), MNCN (Museo Nacional de Ciencias Naturales, Madrid), AKM (Museo Alexander Koenig, Bonn), USNM (Museo Nacional de Historia Natural, Washington DC). Significado de las abreviaturas utilizadas para los tipos de citas recogidas: $\mathrm{C}=$ Captura, $\mathrm{O}=$ Observación, $\mathrm{CT}=$ Cita sin especificar, $\mathrm{MO}=$ Múltiples observaciones, $\mathrm{MC}=$ Múltiples capturas, $\mathrm{MOC}=$ Múltiples observaciones y capturas, EM = Ejemplar encontrado muerto, $E G=C$ ráneo encontrado en egagrópila, $L U$ = restos encontrados en excremento de nutria Lutra lutra, $C+E X=$ Capturas y excrementos de desmán, $C+I G=$ Captura y excrementos de desmán con identificación genética.

Table 2. Records of occurrence of Iberian desman in the Central System. Collections consulted for recording desman localities: EBD (Doñana Biological Station, Seville), MNCN (National Museum of Natural Sciences, Madrid), AKM (Alexander Koenig Museum, Bonn), USNM (National Museum of Natural History, Washington DC). Meaning of the abbreviations used to indicate the kind of citation recorded: $\mathrm{C}=$ Capture, $\mathrm{O}=\mathrm{Observation}, \mathrm{CT}=$ Citation with non specified origin, $\mathrm{MO}=$ Multiple observations, $\mathrm{MC}=$ Multiple captures, $\mathrm{MOC}=$ Multiple observations and captures, $\mathrm{EM}=$ Specimen found dead. $\mathrm{EG}=$ Skull found in owl pellet, $L U=$ remains found in otter feces $L$ utra lutra, $C+E X=$ Captures and desman feces, $C+I G=$ Capture and desman feces with genetic identification . 


\begin{tabular}{|c|c|c|c|c|}
\hline Localidad/Río & Término Municipal & Fecha & Fuente & Tipo de cita \\
\hline \multicolumn{5}{|l|}{ GUADALAJARA } \\
\hline Río Jaramilla & Peñalba de la Sierra & 11995 & Gisbert y Fernández-Salvador (1998) & $\mathrm{N}+\mathrm{ED}+\mathrm{EC}$ \\
\hline Río de la Hoz & Cantalojas & 1995 & Gisbert y Fernández-Salvador (1998) & $\mathrm{N}+\mathrm{ED}+\mathrm{EC}$ \\
\hline Río Berbellido & Bocígano & 1996 & Gisbert y Fernández-Salvador (1998) & $\mathrm{N}+\mathrm{ED}+\mathrm{EC}$ \\
\hline Río de la Zarza & Cantalojas & 1996 & Gisbert y Fernández-Salvador (1998) & $\mathrm{N}+\mathrm{ED}+\mathrm{EC}$ \\
\hline Río de la Zarza & Cantalojas & 1996 & Gisbert y Fernández-Salvador (1998) & $\mathrm{N}+\mathrm{ED}+\mathrm{EC}$ \\
\hline Río Lillas & Cantalojas & 1996 & Gisbert y Fernández-Salvador (1998) & $\mathrm{N}+\mathrm{ED}+\mathrm{EC}$ \\
\hline Río Sonsaz & Cantalojas & 1996 & Gisbert y Fernández-Salvador (1998) & $\mathrm{N}+\mathrm{ED}+\mathrm{EC}$ \\
\hline Río Sorbe & Valverde del Arroyo & 1996 & Gisbert y Fernández-Salvador (1998) & $\mathrm{N}+\mathrm{ED}+\mathrm{EC}$ \\
\hline Río Dulce & Pelegrina & 1996 & Gisbert y Fernández-Salvador (1998) & $\mathrm{N}+\mathrm{ED}+\mathrm{EC}$ \\
\hline Río Dulce & Pelegrina & 1996 & Gisbert y Fernández-Salvador (1998) & $\mathrm{N}+\mathrm{ED}+\mathrm{EC}$ \\
\hline Río Sorbe & Cantalojas & antes de 1992 & Nores (1992) & $\mathrm{N}+\mathrm{ED}+\mathrm{EC}$ \\
\hline Río Sorbe & Galve de Sorbe & antes de 1993 & Nores (1992) & $\mathrm{N}+\mathrm{ED}+\mathrm{EC}$ \\
\hline Río Lillas & Cantalojas & antes de 1994 & Nores (1992) & $\mathrm{N}+\mathrm{ED}+\mathrm{EC}$ \\
\hline \multicolumn{5}{|l|}{ SEGOVIA } \\
\hline Río Eresma & Balsaín & antes de 1992 & Nores (1992) & $E D+E C$ \\
\hline Río Moros & El Espinar & antes de 1992 & Nores (1992) & $E D+E C$ \\
\hline Río Duratón & Siguero & antes de 1992 & Nores (1992) & $\mathrm{ED}+\mathrm{EC}$ \\
\hline Río Serrano & Cerezo de Arriba & antes de 1992 & Nores (1992) & $\mathrm{ED}+\mathrm{EC}$ \\
\hline Río Serrano & Castillejo de Mesleón & antes de 1992 & Nores (1992) & $E D+E C$ \\
\hline Río Riaza & Riofrío de Riaza & antes de 1992 & Nores (1992) & $\mathrm{ED}+\mathrm{EC}$ \\
\hline Río Riaza & Riaza & antes de 1992 & Nores (1992) & $\mathrm{ED}+\mathrm{EC}$ \\
\hline Arroyo del Puerto & San IIdefonso & 2006 & Regis et al. (2006) & ED \\
\hline Arroyo Minguete & San Ildefonso & 2006 & Regis et al. (2006) & ED \\
\hline Rio de la Acebeda & San Ildefonso & 2006 & Regis et al. (2006) & ED \\
\hline Rio Cambrones & San Ildefonso & 2006 & Regis et al. (2006) & ED \\
\hline Artiñuela y afluentes & Navafría & 2006 & Regis et al. (2006) & ED \\
\hline Riaza & Ríofrio de Riaza & 2006 & Regis et al. (2006) & ED \\
\hline Arroyo Chorrera & Riaza & 2006 & Regis et al. (2006) & ED \\
\hline Río Moros & El Espinar & 2006 & García \& Lizana (2007) & $\mathrm{ED}+\mathrm{EC}$ \\
\hline Río Eresma & Boca del Asno, S. Ildefonso & 2006 & García \& Lizana (2007) & $E D+E C$ \\
\hline Arroyo de Peñalara & San Ildefonso & 2006 & García \& Lizana (2007) & $\mathrm{ED}+\mathrm{EC}$ \\
\hline Río de las Pozas & Aldealengua de Pedraza & 2006 & García \& Lizana (2007) & $\mathrm{ED}+\mathrm{EC}$ \\
\hline Arroyo Peña Negra & Gallegos & 2006 & García \& Lizana (2007) & $\mathrm{ED}+\mathrm{EC}$ \\
\hline Río Prádena & Prádena & 2006 & García \& Lizana (2007) & $\mathrm{ED}+\mathrm{EC}$ \\
\hline \multicolumn{5}{|l|}{ MADRID } \\
\hline Río Jarama & Montejo de la Sierra & 2001 & García-Perea et al. (2001) & $\mathrm{ED}+\mathrm{EC}$ \\
\hline Arroyo La Angostura & Rascafria & 2001 & García-Perea et al. (2001) & $\mathrm{ED}+\mathrm{EC}$ \\
\hline Río Lozoya & Rascafria & 2001 & García-Perea et al. (2001) & $\mathrm{ED}+\mathrm{EC}$ \\
\hline Río Madarquillos & Horcajo de la Sierra & 2001 & García-Perea et al. (2001) & $\mathrm{ED}+\mathrm{EC}$ \\
\hline Río Madarquillos & Piñuecar & 2001 & García-Perea et al. (2001) & $\mathrm{ED}+\mathrm{EC}$ \\
\hline Río de la Garita & Horcajuelo de la Sierra & 2001 & García-Perea et al. (2001) & $\mathrm{ED}+\mathrm{EC}$ \\
\hline Río Jarama & Montejo de la Sierra & 2001 & García-Perea et al. (2001) & $\mathrm{ED}+\mathrm{EC}$ \\
\hline Río de la Puebla & Puebla de la Sierra & 2001 & García-Perea et al. (2001) & $\mathrm{ED}+\mathrm{EC}$ \\
\hline Río Cofio & Valdemaqueda & 2001 & García-Perea et al. (2001) & $\mathrm{ED}+\mathrm{EC}$ \\
\hline Río Canencia & Canencia & 2001 & García-Perea et al. (2001) & $\mathrm{ED}+\mathrm{EC}$ \\
\hline Río Aceña & Santa Mª de la Alameda & 2001 & García-Perea et al. (2001) & $\mathrm{ED}+\mathrm{EC}$ \\
\hline Río Aceña & Estación de El Pimpollar & 2001 & García-Perea et al. (2001) & $\mathrm{ED}+\mathrm{EC}$ \\
\hline Río Santa Ana & Rascafría & 2001 & García-Perea et al. (2001) & $\mathrm{ED}+\mathrm{EC}$ \\
\hline Río Lozoya & Rascafría & 1979 & G. Álvarez, com. pers. & $\mathrm{N}$ \\
\hline Río Lozoya & Pinilla del Valle, Rascafría & $1980-1981$ & B. Ramos, com. pers. & $\mathrm{N}$ \\
\hline Río Jarama & Montejo de la Sierra & $<1992$ & Nores (1992) & $\mathrm{ED}+\mathrm{EC}$ \\
\hline Río Garcisancho & Rascafría & $<1992$ & Nores (1992) & $\mathrm{ED}+\mathrm{EC}$ \\
\hline Río Navalmedio & Cercedilla & $<1992$ & Nores (1992) & $\mathrm{ED}+\mathrm{EC}$ \\
\hline \multicolumn{5}{|l|}{ ÁVILA } \\
\hline Garganta del Endrinal & Mazalinos, Solana de Ávila & 2000 & Gisbert et al. (2000) & $\mathrm{N}+\mathrm{ED}+\mathrm{EC}$ \\
\hline Garganta de Solana & Solana de Ávila & 2000 & Gisbert et al. (2000) & $\mathrm{N}+\mathrm{ED}+\mathrm{EC}$ \\
\hline
\end{tabular}




\begin{tabular}{|c|c|c|c|c|}
\hline \multicolumn{5}{|l|}{ ÁVILA } \\
\hline Río Aravalle & Puerto Castilla & 2000 & Gisbert et al. (2000) & $\mathrm{N}+\mathrm{ED}+\mathrm{EC}$ \\
\hline Garganta Barbellido & Navacepeda de Tormes & 2000 & Gisbert et al. (2000) & $\mathrm{N}+\mathrm{ED}+\mathrm{EC}$ \\
\hline Río Tormes & Hoyos del Espino & 2000 & Gisbert et al. (2000) & $\mathrm{N}+\mathrm{ED}+\mathrm{EC}$ \\
\hline Río Tormes & La Aliseda de Tormes & 2000 & Gisbert et al. (2000) & $\mathrm{N}+\mathrm{ED}+\mathrm{EC}$ \\
\hline Garganta de la Nava & La Nava del Barco & 2000 & Gisbert et al. (2000) & $\mathrm{N}+\mathrm{ED}+\mathrm{EC}$ \\
\hline Garganta de Gredos & Navalperal de Tormes & 2000 & Gisbert et al. (2000) & $\mathrm{N}+\mathrm{ED}+\mathrm{EC}$ \\
\hline Regadera Angostura & La Angostura & 2000 & Gisbert et al. (2000) & $\mathrm{N}+\mathrm{ED}+\mathrm{EC}$ \\
\hline Garganta Caballeros & Navalguijo, Navalonguilla & 2000 & Gisbert et al. (2000) & $\mathrm{N}+\mathrm{ED}+\mathrm{EC}$ \\
\hline Garganta de Bohoyo & Bohoyo & 2000 & Gisbert et al. (2000) & $\mathrm{N}+\mathrm{ED}+\mathrm{EC}$ \\
\hline Garganta de Iruelas & El Barraco & 2000 & Gisbert et al. (2000) & $\mathrm{N}+\mathrm{ED}+\mathrm{EC}$ \\
\hline Río Tormes & Hoyos del Espino & $<1992$ & Nores (1992) & $\mathrm{N}+\mathrm{ED}$ \\
\hline Río Tormes & Zapardiel de la Ribera & $<1992$ & Nores (1992) & $\mathrm{N}+\mathrm{ED}$ \\
\hline Río Tormes & Navacepeda de Tormes & $<1992$ & Nores (1992) & $\mathrm{N}+\mathrm{ED}$ \\
\hline Río Corneja & Mesegar de Corneja & $<1992$ & Nores (1992) & $\mathrm{N}+\mathrm{ED}$ \\
\hline Garganta San Miguel & Navalonguilla & $<1992$ & Nores (1992) & $\mathrm{N}+\mathrm{ED}$ \\
\hline Río Alberche & Navaluenga & $<1992$ & Nores (1992) & $\mathrm{N}+\mathrm{ED}$ \\
\hline Río Piquillo & Puerto del Pico, Villarejo del Valle & $<1992$ & Nores (1992) & $\mathrm{N}+\mathrm{ED}$ \\
\hline Río Arenal & El Arenal & $<1992$ & Nores (1992) & $\mathrm{N}+\mathrm{ED}$ \\
\hline Garganta Sta. María & Candeleda & $2008-2009$ & Fernández-González (2009-2010) & $E D+I G$ \\
\hline Garganta de Chilla & Candeleda & $2008-2009$ & Fernández-González (2009-2010) & $E D+I G$ \\
\hline Ggta. del río Muelas & Arenas de San Pedro & $2008-2009$ & Fernández-González (2009-2010) & $E D+I G$ \\
\hline Ggta. del río Arbillas & Arenas de San Pedro & $2008-2009$ & Fernández-González (2009-2010) & $E D+I G$ \\
\hline Río Pelayos & Guisando & $2008-2009$ & Fernández-González (2009-2010) & $E D+I G$ \\
\hline \multicolumn{5}{|l|}{ CÁCERES } \\
\hline Arroyo Santihervás & Hervás & 2003 & Gisbert y García-Perea (2004a) & $\mathrm{N}+\mathrm{ED}+\mathrm{EC}$ \\
\hline Garganta Beceda & Tornavacas & 2003 & Gisbert y García-Perea (2004a) & $\mathrm{N}+\mathrm{ED}+\mathrm{EC}$ \\
\hline Ggta. de San Martín & Tornavacas & 2003 & Gisbert y García-Perea (2004a) & $\mathrm{N}+\mathrm{ED}+\mathrm{EC}$ \\
\hline Garganta de La Serrá & Tornavacas & 2003 & Gisbert y García-Perea (2004a) & $\mathrm{N}+\mathrm{ED}+\mathrm{EC}$ \\
\hline Ggta. Los Asperones & Tornavacas & 2003 & Gisbert y García-Perea (2004a) & $\mathrm{N}+\mathrm{ED}+\mathrm{EC}$ \\
\hline Ggta. Collado Yeguas & Jerte & 2003 & Gisbert y García-Perea (2004a) & $\mathrm{N}+\mathrm{ED}+\mathrm{EC}$ \\
\hline Ggta. de los Infiernos & Jerte & 2003 & Gisbert y García-Perea (2004a) & $\mathrm{N}+\mathrm{ED}+\mathrm{EC}$ \\
\hline Garganta Mayor & Garganta La Olla & 2003 & Gisbert y García-Perea (2004a) & $\mathrm{N}+\mathrm{ED}+\mathrm{EC}$ \\
\hline Garganta Jaranda & El Guijo de Santa Bárbara & 2003 & Gisbert y García-Perea (2004a) & $\mathrm{N}+\mathrm{ED}+\mathrm{EC}$ \\
\hline Garganta del Viento & El Guijo de Santa Bárbara & 2003 & Gisbert y García-Perea (2004a) & $\mathrm{N}+\mathrm{ED}+\mathrm{EC}$ \\
\hline Ggta. del Campanario & El Guijo de Santa Bárbara & 2003 & Gisbert y García-Perea (2004a) & $\mathrm{N}+\mathrm{ED}+\mathrm{EC}$ \\
\hline Garganta de Cuartos & El Losar de la Vera & 2003 & Gisbert y García-Perea (2004a) & $\mathrm{N}+\mathrm{ED}+\mathrm{EC}$ \\
\hline Ggta. del Cogorzal & El Losar de la Vera & 2003 & Gisbert y García-Perea (2004a) & $N+E D+E C$ \\
\hline Ggta. del Escobarejo & El Losar de la Vera & 2003 & Gisbert y García-Perea (2004a) & $\mathrm{N}+\mathrm{ED}+\mathrm{EC}$ \\
\hline Garganta de la Hoz & El Losar de la Vera & 2003 & Gisbert y García-Perea (2004a) & $\mathrm{N}+\mathrm{ED}+\mathrm{EC}$ \\
\hline Arroyo Covachuelas & El Losar de la Vera & 2003 & Gisbert y García-Perea (2004a) & $\mathrm{N}+\mathrm{ED}+\mathrm{EC}$ \\
\hline Arroyo Vahillo & El Losar de la Vera & 2003 & Gisbert y García-Perea (2004a) & $\mathrm{N}+\mathrm{ED}+\mathrm{EC}$ \\
\hline Ggta. de Río Moros & Viandar de la Vera & 2003 & Gisbert y García-Perea (2004a) & $\mathrm{N}+\mathrm{ED}+\mathrm{EC}$ \\
\hline Ggta. de Gualtaminos & Villanueva de la Vera & 2003 & Gisbert y García-Perea (2004a) & $\mathrm{N}+\mathrm{ED}+\mathrm{EC}$ \\
\hline Ggta. de Minchones & Villanueva de la Vera & 2003 & Gisbert y García-Perea (2004a) & $\mathrm{N}+\mathrm{ED}+\mathrm{EC}$ \\
\hline G. Hoz de Villanueva & Villanueva de la Vera & 2003 & Gisbert y García-Perea (2004a) & $\mathrm{N}+\mathrm{ED}+\mathrm{EC}$ \\
\hline Garganta del Hornito & Madrigal de la Vera & 2003 & Gisbert y García-Perea (2004a) & $\mathrm{N}+\mathrm{ED}+\mathrm{EC}$ \\
\hline Garganta de Alardos & Madrigal de la Vera & 2003 & Gisbert y García-Perea (2004a) & $\mathrm{N}+\mathrm{ED}+\mathrm{EC}$ \\
\hline Garganta Jaranda & Jarandilla de la Vera & $<1992$ & Nores (1992) & $\mathrm{N}+\mathrm{ED}$ \\
\hline Río Jerte & Tornavacas & $<1992$ & Nores (1992) & $\mathrm{N}+\mathrm{ED}$ \\
\hline Río Jerte & El Rebollar & $<1992$ & Nores (1992) & $\mathrm{N}+\mathrm{ED}$ \\
\hline Río Ambroz & Hervás & $<1992$ & Nores (1992) & $\mathrm{N}+\mathrm{ED}$ \\
\hline Río Ambroz & Segura de Toro & $<1992$ & Nores (1992) & $\mathrm{N}+\mathrm{ED}$ \\
\hline Río Ambroz & Casas del Monte & $<1992$ & Nores (1992) & $\mathrm{N}+\mathrm{ED}$ \\
\hline Río Arrago & Robledillo de Gata & 2006 & Gisbert (2006) & ED \\
\hline Río Ladrillar & Ladrillar & 2006 & Gisbert (2006) & ED \\
\hline Río Ambroz & Zarza de Granadilla & $<1992$ & Nores (1992) & $\mathrm{N}+\mathrm{ED}$ \\
\hline \multicolumn{5}{|l|}{ SALAMANCA } \\
\hline Río Cuerpo de Hombre & Candelario & $<1992$ & Nores (1992) & $\mathrm{N}+\mathrm{ED}$ \\
\hline
\end{tabular}




\begin{tabular}{|c|c|c|c|c|}
\hline \multicolumn{5}{|l|}{ SALAMANCA } \\
\hline Río Cuerpo de Hombre & Lagunilla & $<1992$ & Nores (1992) & $\mathrm{N}+\mathrm{ED}$ \\
\hline Río Frío & Villasrubias & 2007 & Fernández González (2007) & $\mathrm{ED}+\mathrm{EC}$ \\
\hline Río Águeda & Peñaparda & 2007 & Fernández González (2007) & $\mathrm{ED}+\mathrm{EC}$ \\
\hline Río Águeda & El Payo & 2007 & Fernández González (2007) & $\mathrm{ED}+\mathrm{EC}$ \\
\hline Río Águeda & Navasfrías & 2007 & Fernández González (2007) & $\mathrm{ED}+\mathrm{EC}$ \\
\hline Río Mayas & Robleda & 2007 & Fernández González (2007) & $\mathrm{ED}+\mathrm{EC}$ \\
\hline Regato de la Dehesa & Candelario & 2006-2009 & García-Díaz (2012) & $\mathrm{N}+\mathrm{ED}$ \\
\hline Río Cuerpo de Hombre & Candelario & $2006-2010$ & García-Díaz (2012) & $\mathrm{N}+\mathrm{ED}$ \\
\hline Arroyo de Candelario & Candelario & $2006-2010$ & García-Díaz (2012) & $\mathrm{N}+\mathrm{ED}$ \\
\hline Arroyo del Oso & La Hoya-Navacarros & $2006-2010$ & García-Díaz (2012) & $\mathrm{N}+\mathrm{ED}$ \\
\hline Arroyo de la Jarilla & Candelario & $2006-2010$ & García-Díaz (2012) & $\mathrm{N}+\mathrm{ED}$ \\
\hline Arroyo del Oso & La Hoya-Navacarros & $2006-2010$ & García-Díaz (2012) & $\mathrm{N}+\mathrm{ED}$ \\
\hline Río Cuerpo de Hombre & Béjar & $2006-2010$ & García-Díaz (2012) & $\mathrm{N}+\mathrm{ED}$ \\
\hline Río Cuerpo de Hombre & Montemayor del Río & $2006-2010$ & García-Díaz (2012) & $\mathrm{N}+\mathrm{ED}$ \\
\hline Río Cuerpo de Hombre & Béjar & $2007-2010$ & García-Díaz (2012) & $\mathrm{N}+\mathrm{ED}$ \\
\hline Río Cuerpo de Hombre & Béjar & $2007-2010$ & García-Díaz (2012) & $\mathrm{N}+\mathrm{ED}$ \\
\hline Río Alagón & Sotoserrano & $2007-2010$ & García-Díaz (2012) & ED \\
\hline Río Cuerpo de Hombre & Sotoserrano & $2007-2010$ & García-Díaz (2012) & ED \\
\hline Río Francia & Sotoserrano & $2007-2010$ & García-Díaz (2012) & ED \\
\hline Río Francia & Miranda del Castañar & $2008-2010$ & García-Díaz (2012) & ED \\
\hline Arroyo del Cabril & Herguijuela de la Sierra & $2007-2010$ & García-Díaz (2012) & $\mathrm{N}+\mathrm{ED}$ \\
\hline Río Francia & Casas del Conde & $2007-2010$ & García-Díaz (2012) & $\mathrm{N}+\mathrm{ED}$ \\
\hline Río Francia & El Cabaco & $2007-2010$ & García-Díaz (2012) & $\mathrm{N}+\mathrm{ED}$ \\
\hline Arroyo del Lera & La Alberca & $2007-2010$ & García-Díaz (2012) & $\mathrm{N}+\mathrm{ED}$ \\
\hline Río Batuecas & La Alberca & $2007-2010$ & García-Díaz (2012) & ED \\
\hline Arroyo Batuecas & La Alberca & $2007-2010$ & García-Díaz (2012) & ED \\
\hline Río Batuecas & La Alberca & $2007-2010$ & García-Díaz (2012) & ED \\
\hline Río Agadón, tramo alto & Monsagro & $2007-2010$ & García-Díaz (2012) & $\mathrm{N}+\mathrm{ED}$ \\
\hline Río Agadón, tramo bajo & Monsagro & $2007-2010$ & García-Díaz (2012) & $\mathrm{N}+\mathrm{ED}$ \\
\hline Río Morasverdes & El Maillo & $2007-2010$ & García-Díaz (2012) & $\mathrm{N}+\mathrm{ED}$ \\
\hline Río Morasverdes & El Maillo & $2007-2010$ & García-Díaz (2012) & $\mathrm{N}+\mathrm{ED}$ \\
\hline Arroyo Tenebrilla & El Maillo & $2007-2010$ & García-Díaz (2012) & $\mathrm{N}+\mathrm{ED}$ \\
\hline Río Mayas & Robleda & $2007-2010$ & García-Díaz (2012) & ED \\
\hline Río Mayas & Robleda & $2007-2010$ & García-Díaz (2012) & ED \\
\hline Río Frio & Villasrubias & $2007-2010$ & García-Díaz (2012) & $\mathrm{N}+\mathrm{ED}$ \\
\hline Río Frio & Villasrubias & $2007-2010$ & García-Díaz (2012) & $\mathrm{N}+\mathrm{ED}$ \\
\hline Río Frio & Villasrubias & $2007-2010$ & García-Díaz (2012) & $\mathrm{N}+\mathrm{ED}$ \\
\hline Río Peñaparda & Peñaparda & $2007-2010$ & García-Díaz (2012) & ED \\
\hline Río Perosín & Perosín & $2007-2010$ & García-Díaz (2012) & ED \\
\hline Río Agueda & Navasfrías & $2007-2010$ & García-Díaz (2012) & ED \\
\hline Regato de Rubioso & El Payo & $2007-2010$ & García-Díaz (2012) & $\mathrm{N}+\mathrm{ED}$ \\
\hline Regato de Rubioso & El Payo & 2009 & Gisbert y García-Perea (2010e) & $\mathrm{N}+\mathrm{ED}+\mathrm{EC}$ \\
\hline Río Frio & Villasrubias & 2009 & Gisbert y García-Perea (2010e) & $\mathrm{N}+\mathrm{ED}+\mathrm{EC}$ \\
\hline Río Alagón & Sotoserrano & $<1992$ & Nores (1992) & ED \\
\hline
\end{tabular}

Tabla 3. - Muestreos realizados en el Sistema Central español cuyos resultados han mostrado la ausencia de desmán. Significado de las abreviaturas utilizadas para los métodos de muestreo: $\mathrm{N}=$ Nasas, $\mathrm{ED}=$ Excrementos de desmán, $\mathrm{EC}=$ Excrementos de carnívoros (Lutra lutra, Neovison vison), IG = Excrementos de desmán con identificación genética.

Table 3. Surveys carried out in the Spanish Central System showing the absence of Iberian desman. Meaning of the abbreviations used to indicate the methodology of sampling: $\mathrm{N}=$ Modified fish traps, $\mathrm{ED}=$ desman feces, $\mathrm{EC}=$ carnivore feces (Lutra lutra, Neovison vison), IG = desman feces with genetic identification.

\section{Distribución histórica del desmán en el Sistema Cen- tral. Periodo 1838-1989}

Las primeras citas documentadas de la presencia del desmán en el Sistema Central datan de mediados del siglo XIX, en concreto Graells estudió un ejemplar capturado en El Escorial (Madrid, Sierra de Guadarrama) en 1838. En esas fechas, la especie parecía ser abundante en las Sierras de Guadarrama y Gredos, tal y como el pro- pio Graells $(1850,1897)$ afirma “... recorriendo la cordillera Carpetana, que tan curioso animal es en ella más común que en la Pirenaica, y que en algunos sitios es bien abundante, pues lo he visto y cogido en todo el valle del Lozoya, desde la laguna de Peñalara hasta Buitrago, desde Navacerrada, por el Balsaín, hasta en los mismos Jardines reales de la Granja; en todos los arroyos que bajan de Siete-picos, en la Maliciosa, Fuenfría, Guada- 
rrama, San Rafael, el Espinar, Boquerón del Infierno, Cuelga-muros, Pinares-llanos, Navas del Marqués, Sierra de Ávila, Navaluenga, Puerto del Pico y Sierras de Gredos, del Barco, de la Duquesa y de Tornavacas, y en el Escorial lo he observado varias veces en el arroyo del Plantel, por encima de la presa del Romeral, y hasta en la misma Fuente de la Teja..." Martínez y Sáez (1876) indica que la especie (Myogalea pirenaica) es conocida en la Sierra de Gredos como "mizclera". En cuanto a la Sierra de Ayllón y la cabecera del río Henares, las citas más antiguas datan de la década de los 70 (Tabla 2), al igual que las de la Serra da Estrela (Portugal), aportadas por Engels (1972).

Las citas históricas analizadas indican que el desmán ibérico se distribuyó, hasta 1990, a lo largo de todo el Sistema Central (Fig. 4A).

\section{Distribución del desmán en el Sistema Central. Pe- riodo 1990-1999}

La contribución más relevante durante la década de los 90 al conocimiento de la distribución del desmán ibérico en España es la recopilación de citas, unida a un gran esfuerzo prospectivo, realizados por Nores (1992). Gracias a este estudio, se pudo disponer por primera vez de un mapa aproximado de la distribución de la especie en España, que constituye un punto de referencia para estudios sobre la conservación de sus poblaciones. No obstante, Nores (1992) no recoge las numerosas citas de las Sierras de Gredos recopiladas por J. Gisbert entre los años 70 y 80, que más tarde se citaron en Gisbert et al. (2000) (Tabla 2). En el Sistema Central, Nores (1992) seleccionó 70 puntos de muestreo, de los cuales 16 resultaron cauces secos, 6 no pudieron ser prospectados por problemas de acceso, y los 48 puntos restantes dieron resultados negativos.
Durante este periodo, se realizaron algunas prospecciones sistemáticas en amplias zonas, tanto del área portuguesa como la española, configurándose el área de distribución portuguesa (Queiroz et al., 1995) y obteniéndose por primera vez datos de ausencia de la especie (Tabla 3) en zonas donde se había descrito previamente su presencia. Por tanto, se obtienen las primeras evidencias del declive del desmán en el Sistema Central (Fernández-Salvador et al., 1997, 1998b).

Las localidades relativas a las citas de presencia y ausencia de desmán en el Sistema Central durante el periodo 1990-1999 se muestran en el mapa de la Fig. 4B.

\section{Distribución reciente del desmán en el Sistema Cen- tral. Periodo 2000-2011}

Las evidencias de regresión encontradas en el Sistema Central han estimulado la realización de una serie de prospecciones a lo largo de la última década, con el objeto de obtener una imagen más fiel de su estado de conservación. Se han realizado muestreos en la Sierra de Ayllón (Fernández-Salvador \& Gisbert, 2000), en la Sierra de Guadarrama, en su vertiente madrileña (García-Perea et al., 2001) y en su vertiente segoviana (Regis et al., 2006; García \& Lizana, 2007); en las Sierras de Gredos, tanto en su vertiente abulense (Gisbert et al., 2000; Fernández-González, 2010), como en su vertiente salmantina (Fernández-González, 2007) y su vertiente cacereña (Gisbert \& García-Perea, 2004a, 2004b, 2005, 2011). Asimismo, se han muestreado las sierras del sur de Salamanca: Valle del Alagón, Sierra de Francia, Batuecas, Sierra de Las Quilamas, Sierra de Gata y El Rebollar (Peris et al., 1999; Morales et al., 2004), habiéndose dado por extinguida la especie en Salamanca recientemente (García et al., 2009; Lizana et al., 2009; García-Díaz et al., 2010; Gisbert \& García-Perea, 2010e; García-Díaz, 2012).

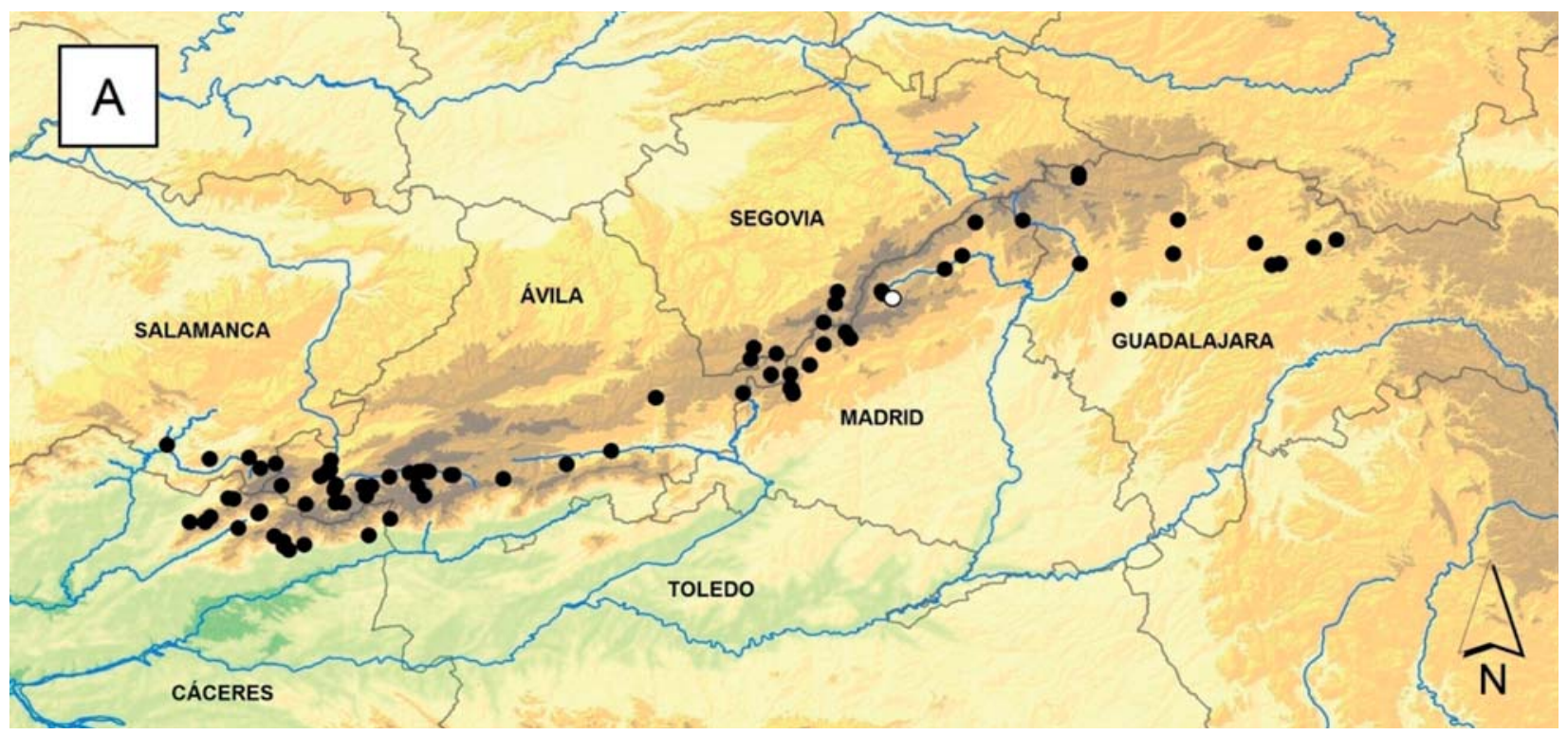



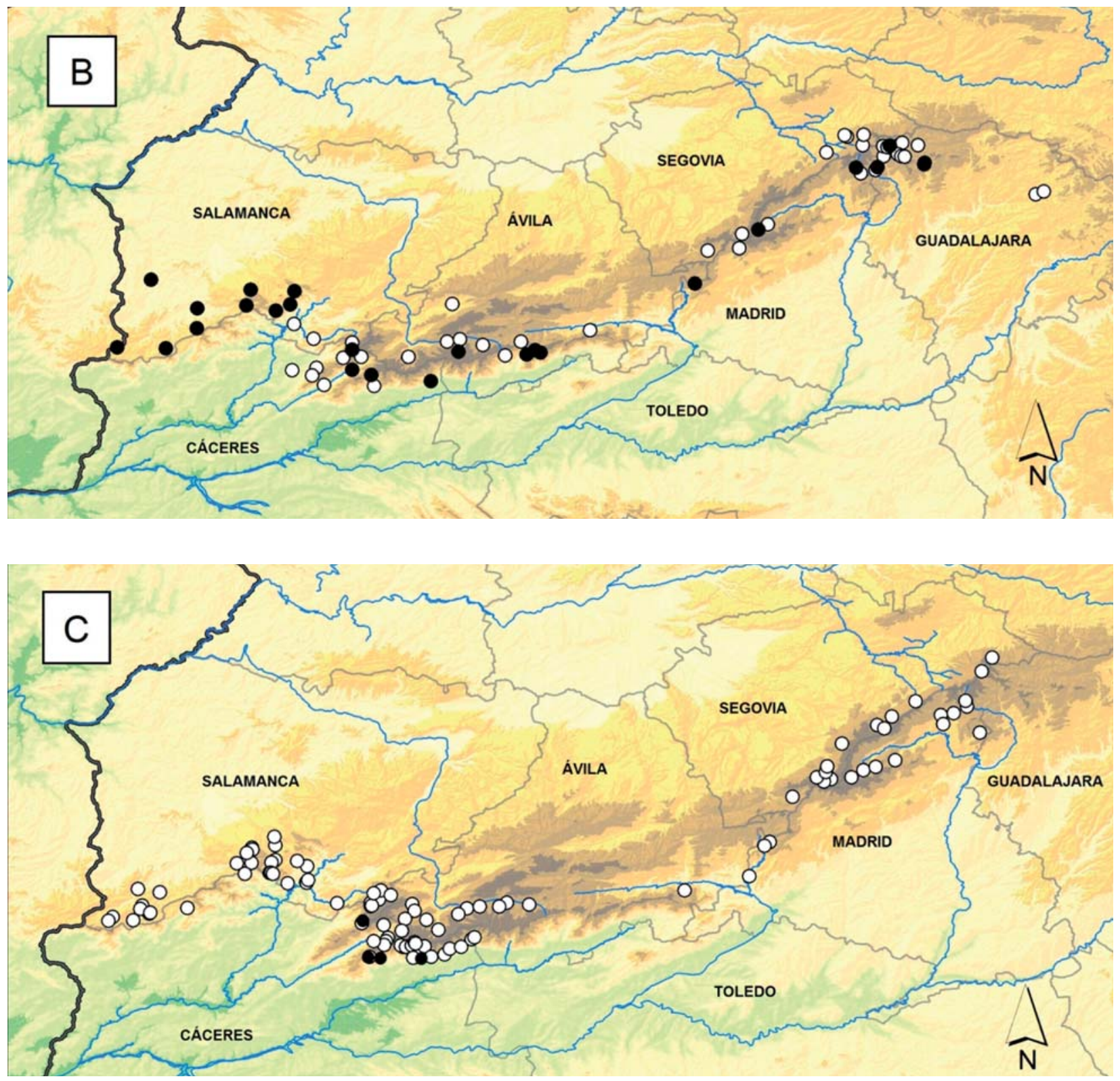

Fig. 4ABC. - Evolución de la distribución del desmán en el Sistema Central, desde las primeras citas históricas hasta los muestreos más recientes. Círculos negros: presencia. Círculos blancos: ausencia. A: Periodo 1838-1989. B: Periodo 1990-1999. C: Periodo 2000-2011.

Fig. 4ABC. - Evolution of the distribution of Iberian desman in the Central System, from the oldest historic reports to the most recent surveys. Black circles: presence. White circles: absence. A: Period 1838-1989. B: Period 1990-1999. C: Period 2000-2011.

Los resultados de este gran esfuerzo de prospección (Tablas 2 y 3) han sido negativos en casi todos los casos, a excepción de tres puntos de presencia en Extremadura y dos en Salamanca (éstas últimas ya desaparecidas). En la actualidad, y con los datos expuestos, podemos decir que posiblemente las únicas poblaciones de desmán que quedan en todo el Sistema Central español son las situadas en tres gargantas de la provincia de Cáceres, en las subcuencas del Alagón y del Tiétar, y en el valle del Jerte.

Las localidades relativas a las citas de presencia y ausencia de desmán en el Sistema Central durante el periodo 2000-2011 se indican en el mapa de la Fig. 4C.

\section{El declive del desmán ibérico en el Sistema Central}

Los resultados expuestos anteriormente clarifican las posibles confusiones creadas por el nombre común "desmán de los Pirineos", que han llevado a algunos autores a dudar de la presencia de la especie en el área de estudio (Burton \& Pearson, 1987; Gosálbez, 1987).

Los datos expuestos también evidencian una gravísima regresión del desmán ibérico en el Sistema Central, que ha ocurrido durante las dos últimas décadas. Las primeras evidencias de este declive se detectaron en la década de los 90 (Fernández-Salvador et al., 1997, 1998b). 
Con las evidencias acumuladas posteriormente (Gisbert et al., 2000, 2001; García-Perea et al., 2001; Gisbert \& García-Perea, 2004a; Gisbert, 2006; Regis et al., 2006; García y Lizana, 2007), Nores (2007) consideró a la especie "En peligro crítico" en el Sistema Central. En 2010, con nuestros resultados más recientes, traspasamos al equipo que trabajaba en la actualización del Catálogo Español de Especies Amenazadas la propuesta de declarar al desmán ibérico "En peligro de Extinción" en el Sistema Central, que fue considerada por el M.A.R.M. en dicho Catálogo (R.D. 139/2011: M.A.R.M., 2011).

La visualización cartográfica de los datos expuestos en los apartados anteriores, nos ha permitido determinar la evolución que han seguido las poblaciones de desmán en el Sistema Central, de acuerdo con los 3 periodos temporales seleccionados (Fig. 4). A continuación, se detallan los datos de regresión de que disponemos para cada una de las áreas del Sistema Central.

- Sierra de Ayllón: Las últimas citas de presencia de desmán en el extremo oriental del Sistema Central corresponden a observaciones en las cabeceras de los ríos Jarama y Sorbe (1988-1990), pero los muestreos realizados en 1995-1996 en esas localidades y otras de la cuenca alta del Henares, arrojaron resultados negativos. No se dispone de datos relativos al proceso de regresión de estas poblaciones.

- Sierra de Guadarrama: 16 citas de presencia en las cabeceras de los ríos Lozoya, Guadarrama y Jarama, de la década de 1980 y principios de los 90, son las últimas de estas sierras, a excepción de una cita del año 2000 en la vertiente segoviana (García \& Lizana, 2007). Pero los numerosos muestreos realizados entre 1979 y 2006 en ambas vertientes de la sierra dieron resultados negativos. Estos datos sugieren que la distribución del desmán en la Sierra de Guadarrama ya estaba fragmentada desde la década de 1970, fecha en la que estimamos el comienzo de la regresión de la especie en dichas sierras.

- Sierras de Gredos: Los últimos datos recogidos sobre el desmán en las Sierras de Gredos son de la década 1991-2000, aunque estos datos son de observaciones puntuales, existiendo numerosos resultados negativos (Tabla 3) de prospecciones sistemáticas realizadas entre 1992-2010. Estos datos son indicadores de un proceso de regresión que ya estaba en marcha en la década de 1990, y aún antes, ya que los estudios continuados de J. Gisbert a lo largo de la década de 1970 y principios de los 80 indican que el comienzo del declive de la especie en esa zona se podría situar a partir de los años 70, década en la que se detectó la invasión de los ríos de Gredos por parte del visón americano, y de forma mucho más acusada a partir del año 1983 en el Alto Tormes (Gisbert et al., 2000).

- Sierras del sur de Salamanca: Según los resultados obtenidos, el desmán estuvo presente en la mayoría de los ríos del sur de Salamanca hasta finales de la década de 1990, existiendo poblaciones en la Sierra de Gata y en las Sierras de Francia y Béjar. En 2007-2009, sólo se encontró desmán en 6 localidades de los ríos Francia y Cuerpo de Hombre, habiendo desaparecido ya de la Sierra de Gata. En 2010, no se pudo constatar ya la presencia del desmán en Salamanca. Estos datos son proporcionados por García-Díaz (2012), quien también identifica dos causas claras en el proceso de extinción del desmán en la porción castellanoleonesa del Sistema Central: la expansión del visón americano y el caudal medio estival de los ríos.

- Serra da Estrela: En la parte portuguesa del Sistema Central, se detectó una disminución de las áreas de presencia de la especie en los años 90 (Queiroz et al., 1998) y, en los últimos sondeos realizados (Quaresma, 2001), los resultados fueron negativos en toda la vertiente sur de la citada sierra. La permanencia de poblaciones más abundantes en el área portuguesa del Sistema Central puede deberse a la influencia atlántica en el clima, que produce unos niveles de precipitación más elevados.

La conclusión de que el desmán está prácticamente desaparecido en el Sistema Central está avalada por el gran esfuerzo de muestreo realizado en el Sistema Central a lo largo de los últimos 15 años, enfocado a la búsqueda de indicios de presencia de la especie o capturas.

\section{Causas de la regresión del desmán en el Sistema Central}

Los datos disponibles sobre la desaparición del desmán en las distintas partes del Sistema Central nos hacen pensar que, más que una causa única, debieron concurrir una serie de factores, la mayoría de carácter antropogénico, cuya actuación simultánea o consecutiva ha originado la regresión de la especie. La información que se ofrece a continuación ha sido expuesta de forma preliminar en varios documentos inéditos, referida a las distintas zonas del Sistema Central estudiadas (Gisbert \& Fernández-Salvador, 1998; Gisbert et al., 2000; García-Perea et al., 2001), pero aquí presentamos un compendio de todos los factores considerados, cuya secuencia y efectos han podido acumularse y ser la causa de la regresión de las poblaciones de desmán ibérico en el Sistema Central:

- 1950-presente. Aumento de la frecuencia de "años secos" durante las últimas 5 décadas (ver, por ejemplo, Benito, 2012). Aunque parece obvio que una especie semiacuática se vea afectada negativamente por una reducción de la pluviosidad, hay que tener en cuenta que el desmán se encontraba en el Sistema Central en una zona climática subóptima, estando su presencia o ausencia determinada por variables relacionadas con la cantidad de precipitación anual y la intensidad de las sequías estivales (Fernández-Salvador et al., 1998a).

- Década de los años 60. Uso generalizado de insecticidas a base de DDT, como consecuencia de los cambios en las actividades agrarias tradicionales. La acumulación de tóxicos vertidos al medio acuático debió afectar letalmente tanto a los propios desmanes, como a sus presas (macroinvertebrados bentónicos). 
- Década de los años 60. Construcción de embalses de agua para consumo humano y regadío. Esto ha tenido varias consecuencias negativas. Por un lado, la alteración de los cauces y los caudales produce cambios en la fauna de macroinvertebrados (pérdida de las condiciones del hábitat requeridas por el desmán). En segundo lugar, los embalses constituyen barreras físicas en los ríos, produciendo la fragmentación de las poblaciones de desmán, e impidiendo su recolonización de los tramos superiores tras fenómenos de desaparición locales.

- 1960-1970. Construcción del alcantarillado, con vertido directo a los ríos sin depurar las aguas, en los pueblos de montaña, resultando en un incremento de la contaminación orgánica y química en las cabeceras de los ríos

- 1960-1975. Aparición y expansión del visón americano. En el Sistema Central, esta invasión parece surgir a partir de granjas peleteras de la Sierra del Guadarrama en la década de los 60, y granjas del sureste de Ávila en la década de los 70. Esta invasión alcanzó Gredos en el año 1975, donde detectamos los primeros ejemplares en el río Tormes. En el extremo oriental, el Visón no parece ser abundante en la Sierra de Ayllón.

- 1970-presente. Dramático incremento estival de la población humana en pueblos de montaña, traduciéndose en una sobre-explotación de los ríos y cursos de agua. Este hecho tiene dos efectos negativos: una reducción brusca de los caudales durante la época estival y un aumento de los vertidos orgánicos a los cauces.

- 1990-2001. Expansión y asentamiento de ciertos depredadores diferentes al visón americano, como la garza real Ardea cinérea Linnaeus, 1758, que en ciertas zonas ha pasado a ocupar durante el verano los mismos lugares de invernada. Esta especie es depredadora ocasional del desmán.

- 1980-presente. Proliferación de azudes, canales y minicentrales hidroeléctricas

- Reducción generalizada del caudal medio de los ríos de montaña (García-Díaz, 2012). Este factor es una combinación de la reducción de la pluviosidad y del abuso en la detracción de agua por parte del hombre. Hay que añadir aquí el abandono del mantenimiento de las redes de acequias y canales de riego, que ha supuesto una disminución de la red hídrica de las comarcas, con la consecuente pérdida de hábitats favorables para el desmán. En la vertiente sur de las Sierras de Gredos, además, se ha producido un incremento en la extracción ilegal de agua por medio de gomas o mangueras, para uso particular, que también contribuye a una reducción de los caudales, sobre todo en el estiaje.

A grandes rasgos, se puede deducir de lo anterior que el desmán pudo sufrir un gran impacto negativo en sus poblaciones del Sistema Central durante la década de los 60, como consecuencia de la contaminación de los ríos y la invasión del visón americano, principalmente. La progresiva reducción y manipulación del caudal de los ríos que ha ocurrido a lo largo de las siguientes décadas, ha impedido la recuperación de las poblaciones y ha provocado su fragmentación, Ilevándolas eventualmente a la desaparición.

\section{Situación actual del desmán ibérico en España}

Como hemos expuesto en los apartados anteriores, nuestros resultados muestran que el desmán ibérico ha sufrido un drástico proceso de declive en el Sistema Central. Se ha estimado que la reducción de su área de distribución en la zona ha sido de un 97\% (Gisbert \& GarcíaPerea, 2010d).

En la actualidad, algunos de los principales factores que han contribuido a la regresión del desmán en el Sistema Central siguen estando vigentes: la expansión y el asentamiento del visón americano, la reducción del caudal de los tramos de montaña y la existencia de barreras que impiden la recolonización de los tramos perdidos, siendo las principales amenazas de la especie en estos momentos (Gisbert \& García-Perea, 2010a). Por estas razones, si no se pone en marcha una estrategia adecuada, para paliar o corregir estas amenazas, es muy probable que el desmán ibérico complete este proceso de regresión y desaparezca del Sistema Central a muy corto plazo.

Paralelamente a este declive en el Sistema Central, se ha producido un declive generalizado en todas las poblaciones de la especie, aunque de menor intensidad, estimado de forma global en un 68\% (Gisbert \& García-Perea, 2010b). Este declive se ha calculado mediante la estima de la superficie perdida de subcuencas con citas históricas fiables, cuyos muestreos recientes han resultado negativos. El mapa de la figura 5 muestra la distribución actual del desmán ibérico en la península Ibérica (Gisbert \& García-Perea, 2010b).

\section{AGRADECIMIENTOS}

Dedicamos esta contribución a nuestros hijos, esperando que en el transcurso de su generación sigan existiendo desmanes en los ríos limpios de la Península Ibérica. P. Cuadrado, S. Hernández, L. Redal, J. Rojo, I. Aguado, I. Carrasco, A. Arilla, M. Sanguino, J. Martínez, A. Antón, M. Bonet, R. Albano, M. Enríquez, R. Fernández, Y. Luque, B. Cobaleda, M.J. Serrano, J.L. Moreno, S. Merayo, E. Cariño y A. Gisbert, formaron parte de los diferentes equipos de campo. J.Ma Fernández-López elaboró los mapas de las figuras 1 y 5. M.J. Serrano elaboró los mapas de las figuras 2, 3 y 4. Agradecemos a C. Rodríguez-Refojos y J. Rubines su invitación a las II Jornadas Técnicas de Mamíferos Semiacuáticos (Vitoria, 2009), de donde surgió la idea de este artículo. Estamos muy agradecidos a los técnicos de las Consejerías de Medio Ambiente de las distintas Comunidades Autónomas que apoyaron nuestros estudios sobre el estatus del Desmán: P. Muñoz, J.M. López Caballero, M.J. Palacios, A. Fernández, Á. Sánchez 


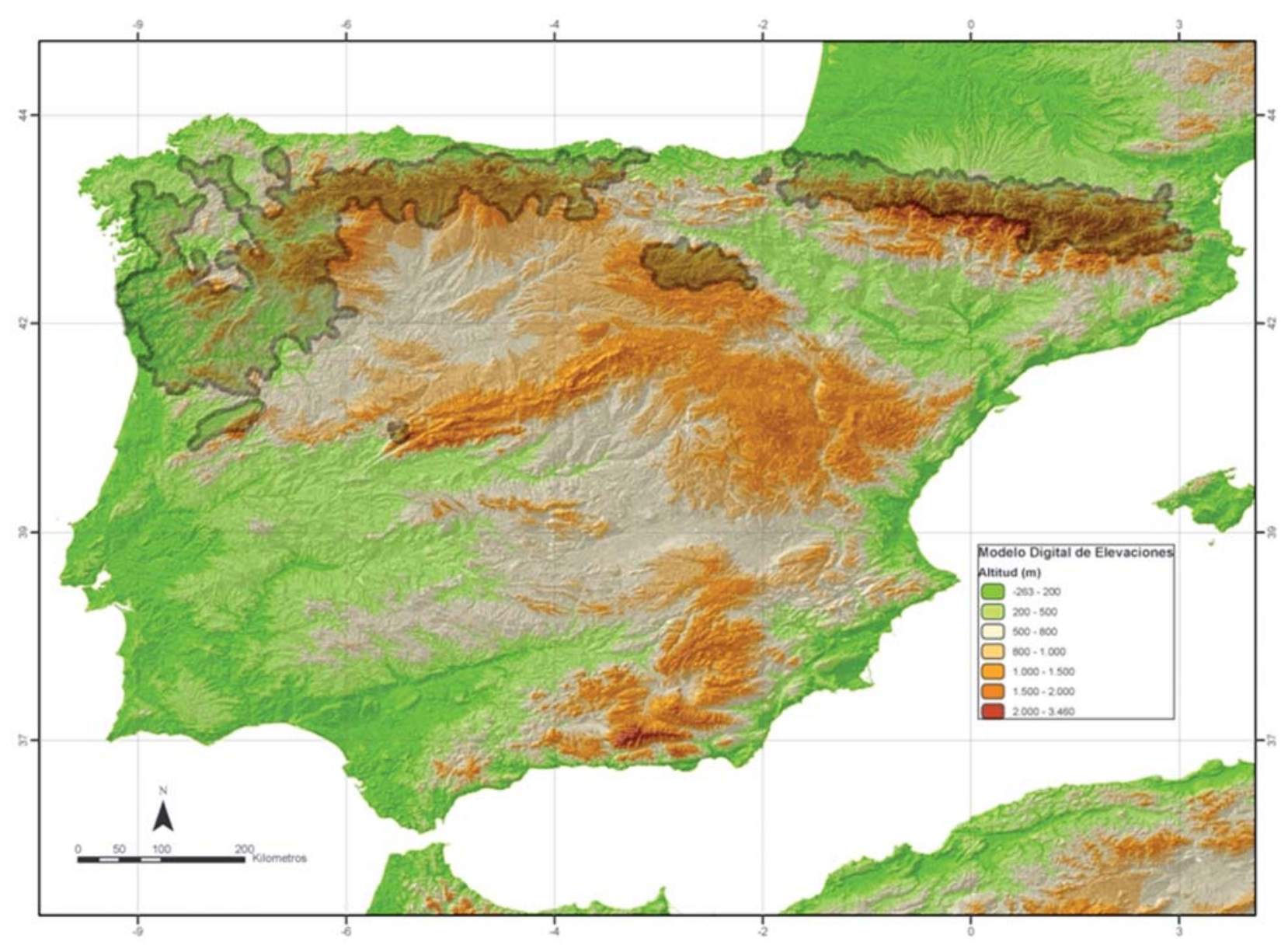

Fig. 5. - Área de distribución actual del desmán ibérico, según Gisbert \& García-Perea (2010b).

Fig. 5. - Present distribution area of Iberian desman, after Gisbert \& García-Perea (2010b).

y A. Gil (Junta de Extremadura); J. Martín y D. Sánchez (Junta de Castilla La Mancha); J.Á. Arranz, I. Molina y César Pollo (Junta de Castilla y León); M. Alcántara (Gobierno de Aragón); L. Prada (Comunidad de Madrid); J. Santamarina (Xunta de Galicia); B. Ramos (Junta de Andalucía). Estamos también agradecidos a G. Alonso, I. Torres y V. Gutiérrez, de la Fundación Biodiversidad, así como a M. Aymerich, R. Gómez Calmaestra, L.M. González, J.R. Areces, B. Heredia y G. Álvarez, del Ministerio de Medio Ambiente. R. Romero, C. Nores, F. Ojeda, M. Lizana, P. García-Díaz y Á. Fernández González aportaron generosamente datos propios para este estudio.

\section{BIBLIOGRAFÍA}

Aymerich, P., Casadesus, F., Gosálbez, J. 2001. Distribució de Galemys pyrenaicus (Insectivora, Talpidae) a Catalunya. Orsis 16: 93-110.

Benito, G. 2012. Incidencia de sequías y catástrofes naturales en la desertización. Crítica 56 (937): 32-36. Disponible en web: http://digital.csic.es/bitstream/10261/81210/1/412604.pdf.

Bertrand, A. 1992. Le desman des Pyrenées, statut, ecologie, conservation. Ministére de l'Environment, Direction de la Protection de la Nature. Rapport inédit.
Bueno, R. 1998. El desmán de los Pirineos (Galemys pyrenaicus, Geoffroy 1811) en la Sierra de Béjar (Sector occidental de Gredos). Galemys 10(1): 49-50.

Burton, J. A., Pearson, B. 1987. Rare Mammals of the World. The Stephen Greene Press.

Cabrera, A. 1912. Catálogo metódico de las Colecciones de Mamíferos del Museo Nacional de Ciencias Naturales de Madrid. Trab. Museo Cienc. Nat. 11: 1-147.

Cabrera, A. 1914. Fauna ibérica. Mamíferos. Museo Nacional de Ciencias Naturales. Madrid.

De la Fuente Arrimadas, N. 1983. Fisiografía e Historia del Barco de Ávila. Excmo. Ayuntamiento de El Barco de Ávila. Edición facsímil de 1983.

Ellis, E.C., Fuller, D.Q., Kaplan, J.O., Lutters, W.G. 2013. Dating the Anthropocene: Towards an empirical global history of human transformation of the terrestrial biosphere. Elementa, Science of the Anthropocene 1(1): 000018. Disponible en web: http://www.elementascience.org/article/info:doi/10.12952/journal.elementa.000018.

Engels, H. 1972. Kleinsäuger aus Portugal. Bonn. Zool. Beitr. 23: $79-86$.

Fernández-González, Á. 2007. Prospección de desmán ibérico (Galemys pyrenaicus) y recogida de excrementos para análisis genético en diversas cuencas de la cornisa cantábrica (Asturias, Cantabria y Castilla y León). BIOSFERA - Laboratori d'Ictiología Genética de la Universitat de Girona. Inédito. 
Fernández-González, Á. 2010. Seguimiento de mamíferos acuáticos (Galemys pyrenaicus y Lutra lutra) en la cuenca del río Tiétar. Medidas compensatorias de la carretera CL - 501: Tramo Candeleda - Ramacastañas (Ávila). ALDESA, Junta de Castilla y León. Inédito.

Fernández-Salvador, R., Gisbert, J., García-Perea, R. 1998a. Biogeography of Galemys pyrenaicus. Euro-American Mammal Congress. Santiago de Compostela, España.

Fernández-Salvador, R., Gisbert, J., García-Perea, R. 1998b. Evidence of decline in the southern border of Galemys pyrenaicus range. Euro-American Mammal Congress. Santiago de Compostela, España.

Fernández-Salvador, R., Gisbert, J., Ojeda, J.F., García-Perea, R., Nores, C. 1997. Indicios de la regresión del desmán ibérico, Galemys pyrenaicus, en el Sistema Central. III Jornadas Españolas de Conservación y Estudio de Mamíferos. Castelló d'Empúries.

García, P., Lizana, M. 2007. Conservación de las poblaciones de vertebrados amenazados y bioindicadores de medios acuáticos del Sistema Central segoviano. Colección Naturaleza y Medio Ambiente. Obra Social y Cultural de Caja Segovia. Segovia.

García, P., Mateos, I., Lizana, M., Vicente, R. 2009. Distribución del Desmán ibérico (Galemys pyrenaicus) en el sur de Salamanca. IX Jornadas de Conservación y Estudio de Mamíferos. Bilbao.

García-Díaz, P. 2012. Situación actual del desmán ibérico (Galemys pyrenaicus) en la provincia de Salamanca. Tesina de Licenciatura. Universidad de Salamanca. Salamanca.

García-Díaz, P., Lizana, M., Mateos, I., Arévalo, V. 2010. El Desmán ibérico Galemys pyrenaicus en la provincia de Salamanca. Documento 6. En: Bases para una estrategia nacional de conservación del desmán ibérico, Galemys pyrenaicus. J. Gisbert, R. García-Perea (Coord.) Fundación Biodiversidad. Inédito.

García-Perea, R., Fernández-Salvador, R., Gisbert, J. 2001. Evaluación del estatus de las poblaciones de Desmán Ibérico, Galemys pyrenaicus, en la vertiente sur de la Sierra de Guadarrama. Comunidad de Madrid. Inédito.

Gisbert, J. 1983. Valoración faunística de las Sierras de Gredos. Proyecto de Investigación 3611 del Instituto Nacional de Investigaciones Agrarias. Inédito.

Gisbert, J. 2006. Informe sobre la situación en España de cuatro especies de micromamíferos amenazados: Galemys pyrenaicus, Sorex granarius, Microtus cabrerae y Chionomys nivalis. Proyecto Inventario Nacional de Biodiversidad. SECEM, Ministerio de Medio Ambiente, TRAGSA. Inédito.

Gisbert, J., Fernández-Salvador, R. 1998. Estudio sobre evaluación de poblaciones de especies de mamíferos, anfibios y reptiles amenazados de Castilla-La Mancha. Bases científicas para su conservación. El Desmán Ibérico, Galemys pyrenaicus. Junta de Castilla-La Mancha. Inédito.

Gisbert, J., García Perea, R. 1988. Los Mamíferos de las Sierras de Gredos. Bol. Universitario (Ávila) 7: 103114.

Gisbert, J., García Perea, R. 2004a. Estudio sobre la distribución de la Almizclera (Galemys pyrenaicus) en Extremadura. Junta de Extremadura. Inédito.

Gisbert, J., García Perea, R. 2004b. Estudio cuantitativo sobre la población de Desmán Ibérico en el valle del Ambroz y Topillo de Cabrera en el norte de Extremadura. Junta de Extremadura. Inédito.

Gisbert, J., García Perea, R. 2005. Estudio y Conservación de Micromamíferos amenazados de Extremadura: El Desmán Ibérico y el Topillo de Cabrera. En: Conservación de la Naturaleza en Extremadura. Comunicaciones en Jornadas y Congresos 2002-2004. J.M. López Caballero (Ed.) Consejería de Agricultura y Medio Ambiente, Junta de Extremadura. Mérida.
Gisbert, J., García Perea, R. 2010a. Catálogo de amenazas del desmán ibérico. Distribución e identificación de núcleos estables. Documento 4. En: Bases para una estrategia nacional de conservación del Desmán ibérico, Galemys pyrenaicus. Actividades 2009/2010. Gisbert, García-Perea (Coord.). Fundación Biodiversidad. Inédito.

Gisbert, J., García Perea, R. 2010b. Diagnóstico 2010 sobre la situación actual del Desmán ibérico en España. Documento 5. En: Bases para una estrategia nacional de conservación del desmán ibérico, Galemys pyrenaicus. Actividades 2009/2010. J. Gisbert, R. García-Perea (Coord.). Fundación Biodiversidad. Inédito.

Gisbert, J., García Perea, R. 2010c. Distribución histórica, regresión de las poblaciones y amenazas del Desmán ibérico. Jornadas Técnicas de Conservación del Desmán ibérico, Galemys pyrenaicus. Zaragoza.

Gisbert, J., García Perea, R. 2010d. La regresión de las poblaciones de Desmán ibérico en España. Distribución e identificación de núcleos estables. Documento 3. En: Bases para una estrategia nacional de conservación del desmán ibérico Galemys pyrenaicus. Actividades 2009/2010. Gisbert, García-Perea (Coord.) Fundación Biodiversidad. Inédito.

Gisbert, J, García Perea, R 2010e. Presencia del Desmán ibérico en los ríos Rubiós y Riofrío, Salamanca. Documento 6. En: Bases para una estrategia nacional de conservación del desmán ibérico, Galemys pyrenaicus. Actividades 2009/2010. J. Gisbert, R. García-Perea (Coord.) Fundación Biodiversidad. Inédito.

Gisbert, J., García-Perea, R. 2011. Comprobación de la presencia de Desmán ibérico (Galemys pyrenaicus) en el río Ambroz (Cáceres). En: Bases para una estrategia nacional de conservación del desmán ibérico, Galemys pyrenaicus. Actividades 2010/2011. J. Gisbert, R. García-Perea (Coord.) Justificación Técnica. Fundación Biodiversidad. Inédito.

Gisbert, J., Fernández-Salvador, R., García-Perea, R. 2000. Estudio sobre la presencia del Desmán Ibérico, Galemys pyrenaicus E. Geoffroy Saint-Hilaire, 1811, en la cara norte de la Sierra de Gredos (Alto Tormes). Junta de Castilla y León. Inédito.

Gisbert, J., Fernández-Salvador, R., García-Perea, R. 2001. Evidencias de regresión en las poblaciones de Desmán Ibérico. Galemys pyrenaicus. V Jornadas Españolas de Conservación y Estudio de Mamíferos. Vitoria.

Gisbert, J., Melendro, J. 1976. La Fauna de Gredos. En: S.O.S. por Gredos. ALBE.

Gosálbez, J. 1987. Insectívors y rosegadors de Catalunya, metodología d'estudi i catàleg faunístic. Editorial Ketres. Barcelona.

Graells, M.P. 1852-1858. Memorias de los trabajos verificados en los años de 1850 a 1855 por la Comisión encargada de formar el Mapa Geológico. Sección Zoológica. Madrid.

Graells, M.P. 1897. Fauna Mastodológica Ibérica. Mem. R. Acad. Esp. Cienc. Exactas, Fis. Nat. 23: 1-806.

Lizana, M., García, P., Mateos, I., Vicente, R. 2009. Distribución del desmán ibérico (Galemys pyrenaicus) en el sur de Salamanca: Libro de Resúmenes. IX Jornadas de la SECEM. Bilbao: 108.

Malo de Molina, J.A., Solano, P. 1989. Mamíferos Insectívoros y Murciélagos de Extremadura. Junta de Extremadura. Inédito.

Martínez y Sáez, R. 1876. Presencia de Myogalea pyrenaica en Gredos. Actas Soc. Esp. Hist. Nat. 5: 23.

Miller, G.S. 1912. Catalogue of the mammals of Western Europe: Europe exclusive of Russia British Museum of Natural History. London.

M.A.R.M. (Ministerio de Medio Ambiente y Medio Rural y Marino). 2011. Real Decreto 139/2011, de 4 de febrero, para el desarrollo del Listado de Especies Silvestres en Régimen de Protección Especial y del Catálogo Español de Especies Amenazadas. BOE 46: 20912-20951. 
Morales, J.J., Lizana, M., Acera, F. 2004. Ecología trófica de la nutria paleártica Lutra lutra en el río Francia (cuenca del Tajo, Salamanca). Galemys 16(2): 57-77.

Niethammer, J. 1956. Insektenfresser und Nager Norspaniens. Bonn. Zool. Beitr. 7: 249-295.

Niethammer, J. 1964. Ein Beitrag zur Kenntnis der Kleinsäuger Nordspaniens. Z. Säugetierkd. 29: 193-220.

Niethammer, G. 1970. Beobachtungen am Pyrenean Desman Galemys pyrenaica. Bonn. Zool. Beitr. 21(3/4): 157-182.

Nores, C. 1992. Aproximación a la metodología y estudio del área de distribución, estatus de población y selección de hábitat del Desmán (Galemys pyrenaicus) en la Península Ibérica. Ministerio de Agricultura, Pesca y Alimentación. Madrid. Inédito.

Nores, C. 2007. Galemys pyrenaicus (E. Geoffroy Saint-Hilaire, 1811) Ficha del Libro Rojo. En: Atlas y Libro Rojo de los Mamíferos Terrestres de España. L.J. Palomo, J. Gisbert, J.C. Blanco (Eds.): 96-98. Dirección General para la Biodiversidad, SECEM, SECEMU. Madrid.

Nores, C., Queiroz, A.I., Gisbert, J. 2002. Galemys pyrenaicus (É. Geoffroy Saint-Hilaire, 1811) Desmán ibérico. En: Atlas de los Mamíferos terrestres de España. L.J. Palomo, J. Gisbert (Eds.): 70-73. Dirección General para la Biodiversidad, SECEM, SECEMU. Madrid.

Nores, C., Queiroz, A.I., Gisbert, J. 2007. Galemys pyrenaicus (É. Geoffroy Saint-Hilaire, 1811) Desmán ibérico. En: Atlas y Libro Rojo de los Mamíferos Terrestres de España. L.J. Palomo, J. Gisbert, J.C. Blanco (Eds.): 92-98. Dirección General para la Biodiversidad, SECEM, SECEMU. Madrid.

Owen-Smith, N. 1999. The Interactions of Humans, Megahervibores, and Habitats in the Late Pleistocene. Extintion Event. In: Extintions in Near Time, Causes, Contexts and Consequences. R.D.E. MacPhee: 57-67. Kluwer Academic/Plenum Publishers. New York.

Peris, S.J., Reyes, E., Hernández, L. 1999. Atlas de los mamíferos silvestres de la provincia de Salamanca. Diputación de Salamanca Ed. Naturaleza y Medio Ambiente.

Quaresma, C.M. 2001. Galemys pyrenaicus: monitoring species occurrence and habitat conservation. $4^{\text {ème }}$ rencontrés sur le desman. Moulis, France.
Queiroz, A.I. 1989. Elementos sobre a distribuição da toupeira-deagua. Actas do I/ Congresso de Areas Protegidas. Lisboa, Portugal. Queiroz, A.I. 1991. Distribution and potential habitat of the Pyrenean Desman (Galemys pyrenaicus Geoffroy, Insectivora, Talpidae) in the "Parque Natural de Montesinho". Arq. Mus. Boc. Nova série 1(27): 385-392.

Queiroz, A.I., Quaresma, C.M., Santos, C.P., Barbosa, A.J., Carvalho, H.M. 1995. Desman (Galemys pyrenaicus) distribution in Portugal, current knowledge. Seminar on the Biology and Conservation of European Desmans and Water Shrews (G. pyrenaicus, D. moschata, Neomys sp.). Ordesa (Espanha): 12-16.

Queiroz, A.I., Quaresma, C.M., Santos, C.P., Barbosa, A.J., Carvalho, H.M. 1998. Bases para a Conservaçao da Toupeira-de-água (Galemys pyrenaicus). Estudios Biol. Conserv. Nat. 27: 1-118.

Regis, Y., Rodríguez, I., Pérez, M. 2006. Distribución del Desmán Ibérico, Galemys pyrenaicus, en la provincia de Segovia. Colección Naturaleza y Medio Ambiente. Caja Segovia. Obra Social y Cultural.

Rey, J.M. 1972. El topo de río. Vida y costumbres de este endemismo de la fauna ibérica. Vida silv. 2: 121-126.

Rey, J.M., Martínez-Rica, J.P. 1977. Dos estudios sobre la fauna de Gredos. Boletín de la C.I.M.A.

Richard, B. 1973. Capture, transport and husbandry of the Pyrenean desman. Int. Zoo Yearb. 13: 174-177.

Romero, R. 2007. Estudio de la dieta del Visón americano (Mustela vison) en Galicia. Posible impacto en las poblaciones de especies amenazadas y de interés especial. Xunta de Galicia. Inédito.

Romero, R. 2010. Detección de la presencia de Desmán Ibérico, en base al análisis de excrementos de Nutria y Visón americano, en Galicia. En: Bases para una estrategia nacional de conservación del desmán Ibérico, Galemys pyrenaicus. Actividades 2009/2010. Asociación Galemia. SIG Rural S.L. Revilla de Camargo (Cantabria). Inédito.

Rumke, C.G. 1985. A review of fossil and recent Desmaninae (Talpidae, Insectivora). Utrecht Micropal. Bull. - Special. Publ. 4.

Toro, M., Granados, I., Aldasoro, J.J., De Hoyo, C., Negro, A., Robles, S., Lizana, M., Morales, J.J. 2001. Las lagunas del Parque Regional de la Sierra de Gredos. Junta de Castilla y León. Valladolid. 